\title{
More Realistic Hamiltonians for the Fractional Quantum Hall Regime in GaAs and Graphene
}

\author{
Michael R. Peterson ${ }^{1,2}$ and Chetan $\operatorname{Nayak}^{2,3}$ \\ ${ }^{1}$ Department of Physics 85 Astronomy, California State University Long Beach, Long Beach, California 90840, USA \\ ${ }^{2}$ Department of Physics, University of California, Santa Barbara, California 93106, USA and \\ ${ }^{3}$ Microsoft Research, Station Q, Elings Hall, University of California, Santa Barbara, California 93106, USA
}

(Dated: November 2, 2018)

\begin{abstract}
We construct an effective Hamiltonian for electrons in the fractional quantum Hall regime for GaAs and graphene that takes into account Landau level mixing (for both GaAs and graphene) and sub-band mixing (for GaAs, due to the non-zero-width of the quantum well). This mixing has the important qualitative effect of breaking particle-hole symmetry as well as renormalizing the strength of the inter-particle interactions. Both effects could have important consequences for the prospect that the fractional quantum Hall effect at $\nu=5 / 2$ is described by states that support nonAbelian excitations such as the Moore-Read Pfaffian or anti-Pfaffian states. For GaAs, Landau level and sub-band mixing break particle-hole symmetry in all Landau levels and sub-band mixing, due to finite-thickness, causes additional short-distance softening of the Coulomb interaction, further renormalizing the Hamiltonian-additionally, the Landau level and sub-band energy spacings are comparable so it is crucial to consider both effects simultaneously. We find that in graphene, Landaulevel mixing only breaks particle-hole symmetry outside of the lowest Landau level $(N \neq 0)$. Landau level mixing is likely to be especially important in graphene since the Landau-level mixing parameter is independent of the external magnetic field and is of order one. Our realistic Hamiltonians will serve as starting points for future numerical studies.
\end{abstract}

PACS numbers: 71.10.Pm, 71.10.+a, 73.43.Cd

\section{INTRODUCTION AND MOTIVATION}

One of the outstanding experimental challenges in physics is to determine whether the fractional quantum Hall effect supports non-Abelian anyon excitations. An affirmative answer would constitute the discovery of a new type of, so far unobserved, particle and could pave the way for topologically-protected quantum information processing $g^{1 / 3}$.

The most promising and notable system for which the search for non-Abelian anyons is taking place is in the $\nu=5 / 2$ fractional quantum Hall effect 4 14 14 The search is motivated by two conjectures. The first is that the Moore-Read Pfaffian state ${ }^{\overline{15}}$ and the anti-Pfaffian state $^{16 \mid 17}$ are representatives of universality classes that have non-Abelian anyon excitations. This conjecture has been recently shown to be true ${ }^{18}$ (see also Refs. [1930). The second conjecture is that the experimentally observed state of matter responsible for the $\nu=5 / 2$ fractional quantum Hall effect (FQHE) is in one of these two universality classes. Several numerical studies ${ }^{31 / 39}$ have provided evidence supporting this conjecture by showing that the ground states of simplified model Hamiltonians are in these two universality classes (Moore-Read Pfaffian or anti-Pfaffian). Even though these model Hamiltonians are physically reasonable, a number of studies $32|34| 35 \mid 38$ highlight the sensitivity of numerical results $31+35 \mid 37$ 39 to the parameters of these 'toy model' Hamiltonians (and to some degree, the system size). Experiments have found that relatively minor modifications to the system such as transposition of the half-filled Landau level from $\nu=5 / 2$ to $\nu=9 / 2,11 / 2, \ldots$ or to $\nu=1 / 2,3 / 2$ lead to metallic states that are anisotropic $\stackrel{40 \mid 41}{4}$ or isotropic and Fermi liquid-like $e^{42}$. Similar anisotropic or isotropic metallic phases are also observed at $\nu=5 / 2$ upon application of, respectively, a small $\stackrel{43 \mid 44}{ }$ or large ${ }^{45}$ in-plane magnetic field (compared in magnitude to the perpendicular magnetic field). Thus, there are at least four different phases (and, perhaps, many more) which can occur at $\nu=5 / 2$, depending on the details of the Hamiltonian. Thus, we are encouraged to ask basic questions which remain unanswered: is the ground state of a more realistic model Hamiltonian for $\nu=5 / 2$ in one of these two non-Abelian universality classes? What is the quantum phase diagram of a realistic Hamiltonian for $\nu=5 / 2$ ?

The sensitivity of the $\nu=5 / 2$ system to small changes in the Hamiltonian is a cause for concern since the canonical simplified model ignores both Landaulevel mixing and the non-zero-width of the quasi-twodimensional (quasi-2D) electron system. (The exceptions are Refs. 37/38, which include Landau-level mixing and Refs. 34 36, which include non-zero-width.) Landau level mixing and non-zero-width can be neglected if the Landau level mixing parameter $\kappa=\left(e^{2} / \epsilon \ell_{0}\right) / \hbar \omega_{c} \ll 1$ and the non-zero-width of the quantum-well $d / \ell_{0} \ll 1$, respectively. Here, $e^{2} / \epsilon \ell_{0}$ is the scale of the Coulomb energy, $\omega_{c}$ is the cyclotron frequency, and $\ell_{0}=\sqrt{\hbar c / e B}$ is the magnetic length, so the dimensionless parameter $\kappa$ is given by $\kappa \approx 2.52 / \sqrt{B[\mathrm{~T}]}$. Therefore, for experiments at magnetic fields in the range $1-10 \mathrm{~T}, \kappa$ is in the range $0.8-2.5$. Similarly, for most GaAs samples $d / \ell_{0} \approx 2-3$. It is not obvious that either one of these parameters can be considered small and, in fact, for common experimental parameters the Landau-level and sub- 
band energy spacing are comparable. Thus, it is potentially dangerous to consider one effect and not the other since, a priori, both effects are of approximately equal importance. Given that the ground state at $\nu=5 / 2$ depends sensitively on the precise Hamiltonian and that the Hamiltonians studied in all previous numerical results neglect potentially important effects, one may question their connection to experiments $46 / 47$.

In fact, recently the FQHE at $\nu=5 / 2$ has been studied experimentally in the regime of strong Landau level mixing $(\kappa>2.5)$ and interesting non-linear behavior of the FQHE energy gap has been observed as a function of $\kappa$ (or density) ${ }^{48}$. Thus, in order to make meaningful experimental predictions for the FQHE in GaAs (or generally any two-dimensional electron gas), it is necessary to study realistic Hamiltonians which include the effects of both Landau level mixing and non-zero-width. This is true not only at $\nu=5 / 2$, but throughout the $N=1$ Landau level, where Landau level mixing is a factor.

The scenario described above for GaAs is potentially an even more pressing issue when one considers the FQHE in graphene. Due to the linear dispersion of electrons in graphene, the cyclotron energy is $\operatorname{sgn}(N) \sqrt{2|N|} \hbar v_{F} / \ell_{0}$, compared to $\hbar \omega(N+1 / 2)$ for GaAs. (In GaAs, $N=0,1,2, \ldots$ whereas in graphene $-\infty<N<\infty)$. This means that the Landau level mixing parameter in graphene $\tilde{\kappa}=\left(e^{2} / \epsilon \ell_{0}\right) /\left(\hbar v_{F} / \ell_{0}\right)=$ $e^{2} /\left(\epsilon \hbar v_{F}\right)$ is, interestingly, independent of magnetic field strength. In addition, the spacing between Landau levels varies as $1 / \sqrt{2 N}$ for large $N$-the Landau levels get closer together in energy the higher up, or lower down below $N=0$, one goes. Consequently, the effective Landau level mixing parameter for the $N^{\text {th }}$ Landau level is $\propto \tilde{\kappa} \sqrt{N}$, which increases with increasing Landau level.

Experimentally, the FQHE has been observed in graphene in suspended samples 4951 (i.e., free standing graphene) and on a boron nitride substrate ${ }^{52}$. In suspended graphene, the Landau level mixing parameter is approximately $\tilde{\kappa} \approx 2.2$ whereas on a boron nitride substrate it is much lower due to the reduction in dielectric constant: $\tilde{\kappa} \approx 0.5-0.8$. In both cases $\tilde{\kappa}$ is obviously not small so it would appear that there is no experimental observation of the FQHE in graphene for which one can ignore Landau level mixing. Many theoretical works have considered the FQHE in graphene ${ }^{53} 58$, pointing out the similarities and differences between the FQHE in GaAs and graphene, but none have taken into account the effect of Landau level mixing.

Therefore, in this paper we construct and study a realistic Hamiltonian for GaAs in the lowest two Landau levels $(N=0$ and $N=1$; no FQHE has been observed in $N \geq 2$ ) that takes into account Landau level and subband mixing. In addition, we construct a realistic Hamiltonian for graphene in the $N=0, \pm 1, \pm 2$ Landau levels which includes Landau level mixing effects which have, so far, been totally ignored. Although we are emphasizing the experimental systems of GaAs and graphene in our calculations, they are appropriate for any fermionic system with either parabolic (GaAs) or linear (graphene) bands up to the specific experimental parameters. Once these effective Hamiltonians are characterized, they can be used to study a variety of problems in which sub-band and/or Landau level mixing effects may play a prominent role.

Our starting point is the systematic treatment of Landau-level mixing formulated in Ref. 59. The effective Hamiltonian for electrons in the $N^{\text {th }}$ Landau level can be derived by integrating out all other Landau levels via an expansion in powers of $\kappa($ or $\tilde{\kappa})$. This was done $e^{59}$ for GaAs for zero width, but the same procedure allows us to integrate out higher sub-bands in the same way as higher Landau levels and/or consider graphene, as we discuss below.

Crucially, we find an error in the normal-ordering of the effective Hamiltonian of the previous work ${ }^{59}$. Correcting this error, we find that the renormalization of the twobody interaction is significantly modified and we examine the implications.

We emphasize that, for GaAs, we include the nonzero width of the $2 \mathrm{D}$ layer, as a result of which the effective interaction between electrons is "softened" at short distances since the single-particle wave functions are smeared out over a length scale $d$ in the direction perpendicular to the plane. This effect was found to stabilize the Moore-Read Pfaffian and anti-Pfaffian states ${ }^{34135}$ in the $N=1$ Landau level, unlike in the lowest Landau level, where it weakens the FQHE. A further effect of non-zero-width is that electron-electron interactions can cause mixing with the higher quantum well sub-bands corresponding to motion in the direction perpendicular to the two-dimensional plane, in a manner analogous to Landau-level mixing. (Strictly speaking, a system with only sub-band mixing could also cause a breaking of particle-hole symmetry. However, Landau-level mixing is generally a stronger effect than sub-band mixing.) We take these two facets of non-zero width (the softening of the "bare" Coulomb interaction and mixing with higher sub-bands) into account in a systematic manner. This has not, to the best of our knowledge, been previously done. Of course, for graphene, the effective width of the $2 \mathrm{D}$ graphene sheet is negligible, so there are no sub-bands that need to be considered.

Strictly speaking, our Hamiltonian can only be justifiably called "realistic" for small values of $\kappa$ (or $\tilde{\kappa}$ ). For GaAs, small $\kappa$ corresponds to higher values of the magnetic field whereas for graphene, small $\tilde{\kappa}$ corresponds to a substrate with high dielectric constant. However, we find that the coefficients in the expansion in powers of $\kappa$ are small in GaAs (even with the correction mentioned in the previous paragraph) and, consequently, our Hamiltonian may even be realistic when $\kappa$ is not small. For graphene, the coefficients in the $N=0$ Landau level are small, similar to those in GaAs, but for $N \neq 0$, this is no longer the case. Thus, these expansions may be valid even in the regime where most experimental observations of the FQHE at $\nu=5 / 2$ in 
GaAs have taken place $(0.7<\kappa<1.8)$, as well as where the FQHE has been experimentally observed in graphene ${ }^{49}[52 / 60](0.5<\tilde{\kappa}<2.2)$. Our calculations might also help explain some of the peculiarities of the graphene FQHE observations, such as the fact that the FQHE has been observed in only the lowest Landau level (see Refs. 49 52). This point will be discussed further below.

Previous results for GaAs: The Landau-level mixing Hamiltonian of Ref. 59 for GaAs was studied for zero width at $\nu=5 / 2$ by Wojs et al $]^{[38}$ using exact diagonalization in the spherical geometry, and it was concluded that, over nearly the entire range of Landau level mixing of experimental interest, $0<\kappa \leq 3$, the overlap between the ground state and the Moore-Read Pfaffian state was larger than either the overlap between the ground state and the composite fermion Fermi-liquid-like wavefunction ${ }^{61]}$ or the overlap between the ground state and the anti-Pfaffian wavefunction. However, the computation of Wojs et al ${ }^{[38}$ did not take finite-thickness of the 2D system into account; it is not clear that these overlaps at different values of the magnetic flux track the (extrapolated) ground state energies, which is what determines the true ground state; and, more importantly, the Hamiltonian did not contain the corrected normalordering of the three-body term. Rezayi and Simon 37 did a similar study using the torus geometry but they did not use the Hamiltonian of Ref. 59 and instead simulated Landau level mixing by diagonalizing in a truncated Hilbert space. In contrast to Wojs et al $\frac{38}{\text {, they }}$ found the anti-Pfaffian to have a higher overlap with the exact ground state. Even though both studies used wavefunction overlap to measure to which universality class the ground state belonged, it is difficult to directly compare their contrasting results since they used different Hamiltonians and different geometries. Of course, it is possible that the previous error in the normal ordering of the three-body term is the origin of these apparently contradictory results.

We point out that there has been additional previous work that considered both sub-band and Landau level mixing 6264 . While many insights can be gained from this work, it is not particularly relevant to this study. For the FQHE, in particular, the effects of both subband and Landau level mixing have been studied, either numerically in the perturbative limit or using a combination of perturbation theory (random phase approximation) and phenomenological models, however, our calculation provides the crucial qualitatively important idea of the breaking of particle-hole symmetry through the generation of three-body terms which were not previously taken into account.

Previous results for graphene: None of the theoretical studies for the FQHE in graphene mentioned above included the effects of Landau level mixing.

The plan of this paper is as follows. In Section II] we review the effective action description given previously in Ref. 59. To this we add the additional complication of a non-zero-width quantum well. This is not as simple as just augmenting the Coulomb interaction because it becomes necessary to integrate out all higher quantum well sub-bands. We then derive the effective Hamiltonian which follows from this effective action, paying particular attention to the operator-ordering of the Hamiltonian. In Sec. III, we discuss the effective Hamiltonian which includes Landau-level and sub-band mixing to first order in the Landau level mixing parameter $\kappa$. In Sec. IV, we consider the effective Hamiltonian for graphene and in Sec. V] we discuss the pseudopotentials. Finally, in Sec. VI, we present our conclusions.

\section{EFFECTIVE HAMILTONIAN INCLUDING LANDAU LEVEL AND SUB-BAND MIXING APPLICABLE TO GaAs}

\section{A. Effective action}

We first begin by discussing the calculation for a system appropriate for GaAs, just to introduce the formalism, and then in Sec. IV we discuss the calculation for a system appropriate to graphene. Following Ref. 59 we begin with the action for electrons in a magnetic field:

$$
\begin{array}{r}
S=\int \frac{d \omega}{2 \pi} \sum_{\text {snm }} \bar{c}_{\text {snmo }}(\omega)\left(i \omega-E_{s n}+\mu\right) c_{s n m \alpha}(\omega) \\
-\frac{1}{2} \int \prod_{i=1}^{4} \frac{d \omega_{i}}{2 \pi} V_{43 ; 21} \bar{c}_{s_{4} n_{4} m_{4} \alpha}\left(\omega_{4}\right) \bar{c}_{s_{3} n_{3} m_{3} \beta}\left(\omega_{3}\right) \\
\times c_{s_{2} n_{2} m_{2} \beta}\left(\omega_{2}\right) c_{s_{1} n_{1} m_{1} \alpha}\left(\omega_{1}\right) \\
\times 2 \pi \delta\left(\omega_{4}+\omega_{3}-\omega_{2}-\omega_{1}\right) .
\end{array}
$$

Here, $c_{\text {snm } \alpha}$ and $\bar{c}_{\text {snm } \alpha}$ are Grassmann variables where $\alpha=\uparrow, \downarrow$ are the spin indices; $s=0,1, \ldots$ is the subband index; $n=0,1, \ldots$ is the Landau level index; and $m=0,1, \ldots$ labels orbital states within a Landau level $\left(m-n\right.$ is the angular momentum). $E_{s n}=$ $\hbar \omega_{c}(n+1 / 2)+\varepsilon_{s}$ is the cyclotron energy $\left(\hbar \omega_{c}=\hbar e B / m c\right)$ plus the sub-band energy $\varepsilon_{s}$, and $\mu$ is the chemical potential. Note that the Zeeman energy has been set to zero in the above action since it is much smaller than the other energy scales in the problem. $V_{43 ; 21} \equiv$ $\left\langle s_{4} n_{4} m_{4}, s_{3} n_{3} m_{3}|\hat{V}| s_{2} n_{2} m_{2}, s_{1} n_{1} m_{1}\right\rangle$ is the interaction matrix element, where $\hat{V}$ is the electron-electron interaction operator for the bare two-body Coulomb interaction for electrons in an infinite quantum well of width, $d$, i.e., the usual non-zero-width augmented interaction investigated previously $\sqrt{34} \sqrt[36 / 65]{70}$. Following the standard procedure ${ }^{71 / 72}$, we define $G_{a b}(k)$ as

$$
G_{a b}(k)=\left(-i \frac{\bar{k}}{\sqrt{2}}\right)^{a-b} \sqrt{\frac{b !}{a !}} L_{b}^{a-b}\left(k^{2} / 2\right)
$$

for integers $a \geq b$ (note that if $a<b$ we switch $k=$ $k_{x}+i k_{y}$ with $\left.\overline{\bar{k}}=k_{x}-i k_{y}\right)$ with $L_{a}^{b}(x)$ a generalized Laguerre polynomial. We find that the interaction matrix 
element is

$$
\begin{aligned}
V_{43,21}= & \int \frac{d^{2} k}{2 \pi} V_{s_{4} s_{3} s_{2} s_{1}}(k) e^{-k^{2}} G_{n_{4} n_{2}}(-\bar{k}) G_{n_{3} n_{1}}(\bar{k}) \\
& \times G_{m_{4} m_{2}}(-k) G_{m_{3} m_{1}}(k),
\end{aligned}
$$

where the 4 in $V_{43,21}$, for example, is short for all the internal degrees of freedom of the fourth particle $\left(s_{4}, n_{4}\right.$, and $\left.m_{4}\right)$ and

$$
\begin{aligned}
V_{s_{4} s_{3} s_{2} s_{1}}(k)= & \frac{e^{2}}{2 \pi \epsilon l_{0}} \frac{1}{k} \int d z_{1} d z_{2} \phi_{s_{4}}\left(z_{1}\right) \phi_{s_{3}}\left(z_{1}\right) \\
& \times \phi_{s_{2}}\left(z_{2}\right) \phi_{s_{1}}\left(z_{2}\right) e^{-k\left|z_{1}-z_{2}\right|},
\end{aligned}
$$

where $\phi_{s}(z)$ is the wavefunction for the $z$-dependence of single-particle wavefunctions in the $s^{\text {th }}$ sub-band. Throughout this work on GaAs we will consider the electrons to be confined to an infinite square well of width $d$. Thus, $\phi_{s}(z)=\sqrt{2 / d} \sin ((s+1) \pi z / d)$ with $z \in[0, d]$ and $\varepsilon_{s}(d)=(s+1)^{2} \pi^{2} \hbar^{2} /\left(2 m_{z} d^{2}\right)$ where $m_{z}$ is the effective electron mass in the quantum well. The total single particle energy is $E_{s n}=\hbar \omega_{c}(n+1 / 2)+\varepsilon_{s}(d)$. If we assume that $m_{z}$ equals the electron band mass $m$ and write $d$ in units of the magnetic length $\ell_{0}=\sqrt{\hbar c / e B} \approx$ $25 \mathrm{~nm} / \sqrt{B[\mathrm{~T}]}$, then $E_{s n}=\hbar \omega_{c}\left[(n+1 / 2)+\left(\pi^{2} / 2 d^{2}\right)(s+\right.$ $\left.1)^{2}\right]$. For typical quantum well widths and magnetic fields $\left(d / \ell_{0} \approx 2-4\right)$ the Landau-level spacing and sub-band spacing are comparable. We further note that the energy spacing between sub-bands gets smaller as the inverse square of the quantum well width unlike the Landau level spacing which is constant. Of course, the potential corresponding to a relevant experimental system is not an infinite square well ${ }^{73 / 74}$, however, the infinite square well captures the key feature of the real potentia $\sqrt{34}-3665$ - 70 : the ability of electrons to avoid each other by moving in the $z$-direction, as reflected both by the softening of the short-distance part of the Coulomb interaction and by the possibility of virtual transitions to higher sub-bands (which have nodes at certain values of $z$ ). We caution the teader that there is one qualitative effect not properly captured by modeling the quantum well with an infinite square well potential; at large enough widths and/or densities, the single layer system can effectively turn into a bilayer. For the purpose of this work, however, we are not considering this situation.

Although the modeling of a more realistic potential is possible within our framework, it is highly dependent on a particular experimental sample. Hence, each sample would require new calculations, which as shown in the following are very laborious. The main purpose of this work, in this regard, is to make general statements and conclusions about the effects of both sub-band and Landau level mixing in a model system. We reiterate, however, that our formalism allows the consideration of more realistic models of quantum wells, through the use of local-density-approximation, for example, in a straightforward manner. These more specific calculations, however, will have to await further study.
We first assume the $N^{\text {th }}$ Landau level to be partially occupied by electrons and integrate out all higher/lower Landau levels and sub-bands perturbatively in the Coulomb interaction. We thereby generate an effective Hamiltonian for the $N^{\text {th }}$ Landau level which incorporates the effects of virtual transitions to the other Landau levels and sub-bands. This effective Hamiltonian is computed perturbatively in powers of $\kappa$, where $\kappa=\left(e^{2} / \epsilon l_{0}\right) /\left(\hbar \omega_{c}\right)$ is the Landau level mixing parameter discussed above $(\kappa \approx 2.52 \times 1 \mathrm{~T} / \sqrt{B[\mathrm{~T}]})$. Thus, $\kappa$ can be varied from very small to very large by changing the magnetic field (and the density, in order to keep the filling fraction fixed). This is is an important distinction between GaAs and graphene, as we discuss in more detail below.

At tree level, the effective action is simply Eq. (1) with the sums over Landau levels restricted to $n_{i}=N$ and the sums over sub-bands restricted to $s_{i}=0$. Most numerical studies of the quantum Hall effect use the effective Hamiltonian associated with this effective action, in which the other Landau levels are integrated out to zeroth order in the Coulomb interaction or, equivalently, to zeroth order in $\kappa$. However, if we integrate out the higher Landau levels to second-order, which amounts to computing the effective action to first-order in $\kappa$, we obtain:

$$
\begin{aligned}
S= & \int \frac{d \omega}{2 \pi} \sum_{m} \bar{c}_{0 N m \alpha}(\omega)\left(i \omega-E_{0 N}+\mu\right) c_{0 N m \alpha}(\omega) \\
- & \frac{1}{2} \int \prod_{i=1}^{4} \frac{d \omega_{i}}{2 \pi} u_{43 ; 21}^{(2)} \bar{c}_{0 N m_{4} \alpha}\left(\omega_{4}\right) \bar{c}_{0 N m_{3} \beta}\left(\omega_{3}\right) \\
& \times c_{0 N m_{2} \beta}\left(\omega_{2}\right) c_{0 N m_{1} \alpha}\left(\omega_{1}\right) 2 \pi \delta\left(\omega_{4}+\omega_{3}-\omega_{2}-\omega_{1}\right) \\
- & \frac{1}{3 !} \int \prod_{i=1}^{6} \frac{d \omega_{i}}{2 \pi} u_{654 ; 321}^{(3)} \bar{c}_{0 N m_{6} \alpha}\left(\omega_{6}\right) \bar{c}_{0 N m_{5} \beta}\left(\omega_{5}\right) \\
& \times \bar{c}_{0 N m_{4} \gamma}\left(\omega_{4}\right) c_{0 N m_{3} \gamma}\left(\omega_{3}\right) c_{0 N m_{2} \beta}\left(\omega_{2}\right) c_{0 N m_{1} \alpha}\left(\omega_{1}\right) \\
& \times 2 \pi \delta\left(\omega_{6}+\omega_{5}+\omega_{4}-\omega_{3}-\omega_{2}-\omega_{1}\right) .
\end{aligned}
$$

As a result of the higher Landau levels which are integrated out, the bare two-body interaction is renormalized

$$
u_{43 ; 21}^{(2)}=V_{43 ; 21}+\kappa \delta u_{43 ; 21}^{(2)}
$$

and a three-body interaction $u_{654 ; 321}^{(3)}$ is generated. At higher-orders in $\kappa$, four-body, five-body, etc., interactions are generated, but we will restrict ourselves here to lowest-order in $\kappa$, so we only need to consider twoand three-body interactions.

The calculation will be presented using Feynman diagrams and the single Coulomb vertex in the diagrammatics is $V_{43,21}^{\beta^{\prime} \alpha^{\prime}, \beta \alpha}=V_{43,21} \delta^{\alpha \alpha^{\prime}} \delta^{\beta \beta^{\prime}}-V_{34,21} \delta^{\alpha \beta^{\prime}} \delta^{\beta \alpha^{\prime}}$ where $\alpha$, $\beta, \alpha^{\prime}$, and $\beta^{\prime}$ label spin indices (see Fig. 1).

We use the nomenclature of Shankar ${ }^{75}$ (as did Ref. (59), (see Fig. 2) for the three diagrams which renormalize the two-body interaction as

$$
\delta u_{43 ; 21}^{(2)} \equiv \mathrm{ZS}+\mathrm{ZS}^{\prime}+\mathrm{BCS}
$$




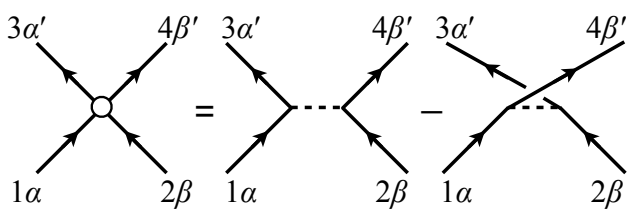

FIG. 1: The bare interaction Feynman diagram. The Coulomb interaction (augmented or not) cannot switch the spin so the first term on the right hand side has $\delta^{\alpha \alpha^{\prime}} \delta^{\beta \beta}$ and the second term has $\delta^{\alpha \beta^{\prime}} \delta^{\beta \alpha^{\prime}}$. The labels $1,2,3$, and 4 correspond to $1=\left\{s_{1}, n_{1}, m_{1}\right\}$, etc..

(a) ZS

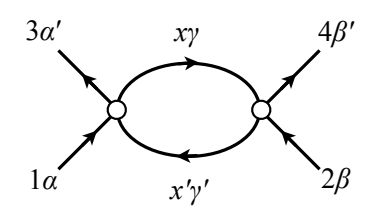

(b) $\mathrm{ZS}^{\prime}$

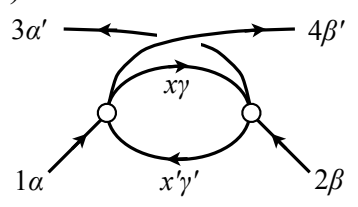

(c) BCS

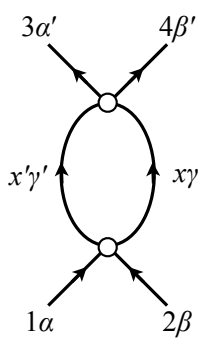

FIG. 2: (a) The ZS Feynman diagram: $V_{x 3, x^{\prime} 1}^{\gamma \alpha^{\prime}, \gamma^{\prime} \alpha} V_{4 x^{\prime}, 2 x}^{\beta^{\prime} \gamma^{\prime}, \beta \gamma}$

The ZS' Feynman diagram: $V_{x 4, x^{\prime} 1}^{\gamma \beta^{\prime}, \gamma^{\prime} \alpha} V_{3 x^{\prime}, 2 x}^{\alpha^{\prime} \gamma^{\prime}, \beta \gamma}$. (c) The BCS Feynman diagram: $V_{43, x x^{\prime}}^{\beta^{\prime} \alpha^{\prime}, \gamma \gamma^{\prime}} V_{x x^{\prime}, 21}^{\gamma \gamma^{\prime}, \beta \alpha}$.

where ZS/ZS' stands for "zero-sound" and BCS stands for "Bardeen-Cooper-Schrieffer" due to their similarity to the corresponding diagrams in Fermi liquid theory. The corresponding expressions are:

$$
\begin{aligned}
\mathrm{ZS} \equiv & \int_{-\infty}^{\infty} \frac{d \omega_{x}}{2 \pi} \int_{-\infty}^{\infty} \frac{d \omega_{x^{\prime}}}{2 \pi} \sum V_{x 3, x^{\prime} 1}^{\gamma \alpha^{\prime}, \gamma^{\prime} \alpha} V_{4 x^{\prime}, 2 x}^{\beta^{\prime} \gamma^{\prime}, \beta \gamma} \\
& \times 2 \pi \delta\left(\omega_{3}+\omega_{x}-\omega_{1}-\omega_{x^{\prime}}\right) G_{x^{\prime}} G_{x} \\
\mathrm{ZS}^{\prime} \equiv & -\int_{-\infty}^{\infty} \frac{d \omega_{x}}{2 \pi} \int_{-\infty}^{\infty} \frac{d \omega_{x^{\prime}}}{2 \pi} \sum V_{x 4, x^{\prime} 1}^{\gamma \beta, \gamma^{\prime} \alpha} V_{3 x^{\prime}, 2 x}^{\alpha^{\prime} \gamma^{\prime}, \beta \gamma} \\
& \times 2 \pi \delta\left(\omega_{x}+\omega_{4}-\omega_{1}-\omega_{x^{\prime}}\right) G_{x^{\prime}} G_{x}
\end{aligned}
$$

and

$$
\begin{aligned}
\mathrm{BCS} \equiv & -\frac{1}{2} \int_{-\infty}^{\infty} \frac{d \omega_{x}}{2 \pi} \int_{-\infty}^{\infty} \frac{d \omega_{x^{\prime}}}{2 \pi} \sum V_{x x^{\prime}, 21}^{\gamma \gamma^{\prime}, \beta \alpha} V_{43, x x^{\prime}}^{\beta^{\prime} \alpha^{\prime}, \gamma \gamma^{\prime}} \\
& \times 2 \pi \delta\left(\omega_{x^{\prime}}+\omega_{x}-\omega_{1}-\omega_{2}\right) G_{x^{\prime}} G_{x}
\end{aligned}
$$

where the electron propagator is $G_{x}=1 /\left(i \omega_{x}-\tilde{E}_{s_{x} n_{x}}\right)$ with $\tilde{E}_{s_{x} n_{x}}=E_{s_{x} n_{x}}-\mu$. We assume the chemical potential to be the energy of the partially filled Landau level, $\mu=\hbar \omega_{c}(N+1 / 2)$, and the above $\Sigma$ is shorthand for the sum

$$
\sum \equiv \sum_{m_{x}, m_{x^{\prime}}=0}^{\infty} \sum_{s_{x}, s_{x^{\prime}}=0}^{\infty} \sum_{n_{x}, n_{x^{\prime}}=0}^{\infty} \sum_{\gamma, \gamma^{\prime}=\downarrow, \uparrow}
$$

Note the primes on the sums over sub-bands $\left(s_{x}\right.$ and $\left.s_{x^{\prime}}\right)$ and Landau levels $\left(n_{x}\right.$ and $\left.n_{x^{\prime}}\right)$ indicate that we do not simultaneously include the conditions $\left(n_{x}=N, s_{x}=0\right)$ or $\left(n_{x^{\prime}}=N, s_{x^{\prime}}=0\right)$-we do not want to integrate out the $N^{\text {th }}$ Landau level of the lowest sub-band.

We now turn to the evaluation of these expressions. Consider the ZS term, for example, and integrate over $\omega_{x^{\prime}}$ using the $\delta$-function:

$$
\begin{aligned}
\mathrm{ZS}= & \int_{-\infty}^{\infty} \frac{d \omega_{x}}{2 \pi} \sum V_{x 3, x^{\prime} 1}^{\gamma \alpha^{\prime}, \gamma^{\prime} \alpha} V_{4 x^{\prime}, 2 x}^{\beta^{\prime} \gamma^{\prime}, \beta \gamma} \\
& \times \frac{1}{i\left(\omega_{3}-\omega_{1}+\omega_{x}\right)-\tilde{E}_{s_{x^{\prime}} n_{x^{\prime}}}} \frac{1}{i \omega_{x}-\tilde{E}_{s_{x} n_{x}}}
\end{aligned}
$$

and we can do the remaining integral over $\omega_{x}$ using the identity

$$
\int_{-\infty}^{\infty} d \omega \frac{1}{i \omega-A} \frac{1}{i \omega-B}=2 \pi \frac{\theta(\operatorname{Re}(A))-\theta(\operatorname{Re}(B))}{B-A}
$$

for $\operatorname{Re}(A) \neq 0$ or $\operatorname{Re}(B) \neq 0$. Thus,

$$
\begin{aligned}
\mathrm{ZS}= & \sum V_{x 3, x^{\prime} 1}^{\gamma \alpha^{\prime}, \gamma^{\prime} \alpha} V_{4 x^{\prime}, 2 x}^{\beta^{\prime} \gamma^{\prime}, \beta \gamma} \\
& \times \frac{\theta\left(\tilde{E}_{s_{x} n_{x}}\right)-\theta\left(E_{s_{x^{\prime}} n_{x^{\prime}}}\right)}{i\left(\omega_{1}-\omega_{3}\right)+\tilde{E}_{s_{x^{\prime}} n_{x^{\prime}}}-\tilde{E}_{s_{x} n_{x}}} .
\end{aligned}
$$

In a similar fashion, we have

$$
\begin{aligned}
\mathrm{ZS}^{\prime}= & -\sum V_{x 4, x^{\prime} 1}^{\gamma \beta, \gamma^{\prime} \alpha} V_{3 x^{\prime}, 2 x}^{\alpha^{\prime} \gamma^{\prime}, \beta \gamma} \\
& \times \frac{\theta\left(\tilde{E}_{s_{x} n_{x}}\right)-\theta\left(\tilde{E}_{s_{x^{\prime}} n_{x^{\prime}}}\right)}{i\left(\omega_{1}-\omega_{4}\right)+\tilde{E}_{s_{x^{\prime}} n_{x^{\prime}}}-\tilde{E}_{s_{x} n_{x}}}
\end{aligned}
$$

and

$$
\begin{aligned}
\mathrm{BCS}= & \frac{1}{2} \sum V_{x x^{\prime}, 21}^{\gamma \gamma^{\prime}, \beta \alpha} V_{43, x x^{\prime}}^{\beta^{\prime} \alpha^{\prime}, \gamma \gamma^{\prime}} \\
& \times \frac{\theta\left(\tilde{E}_{s_{x} n_{x}}\right)-\theta\left(-\tilde{E}_{s_{x^{\prime}} n_{x^{\prime}}}\right)}{i\left(\omega_{1}+\omega_{2}\right)-\tilde{E}_{s_{x^{\prime}} n_{x^{\prime}}}-\tilde{E}_{s_{x} n_{x}}} .
\end{aligned}
$$

We then approximate the denominators as, for example, $i\left(\omega_{1}-\omega_{3}\right)+\tilde{E}_{s_{x^{\prime}} n_{x^{\prime}}}-\tilde{E}_{s_{x} n_{x}} \approx \tilde{E}_{s_{x^{\prime}} n_{x^{\prime}}}-\tilde{E}_{s_{x} n_{x}}$ since $\hbar \omega_{c}$ is much larger than the frequencies at which we probe the system. In principle, however, these effective interactions become retarded if we do not drop the frequency dependence. This approximation eventually yields

$$
\begin{aligned}
\mathrm{ZS} \approx & \sum V_{x 3, x^{\prime} 1}^{\gamma \alpha^{\prime} \gamma^{\prime} \alpha} V_{4 x^{\prime}, 2 x}^{\beta^{\prime} \gamma^{\prime}, \beta \gamma} \\
& \times \frac{\theta\left(\tilde{E}_{s_{x} n_{x}}\right)-\theta\left(\tilde{E}_{s_{x^{\prime}} n_{x^{\prime}}}\right)}{\tilde{E}_{s_{x^{\prime}} n_{x^{\prime}}}-\tilde{E}_{s_{x} n_{x}}},
\end{aligned}
$$




$$
\begin{aligned}
\mathrm{ZS}^{\prime} \approx & -\sum V_{x 4, x^{\prime} 1}^{\gamma \beta, \gamma^{\prime} \alpha} V_{3 x^{\prime}, 2 x}^{\alpha^{\prime} \gamma^{\prime}, \beta \gamma} \\
& \times \frac{\theta\left(\tilde{E}_{s_{x} n_{x}}\right)-\theta\left(\tilde{E}_{s_{x^{\prime}} n_{x^{\prime}}}\right)}{\tilde{E}_{s_{x^{\prime}} n_{x^{\prime}}}-\tilde{E}_{s_{x} n_{x}}},
\end{aligned}
$$

and

$$
\begin{aligned}
\mathrm{BCS} \approx & \frac{1}{2} \sum V_{x x^{\prime}, 21}^{\gamma \gamma^{\prime}, \beta \alpha} V_{43, x x^{\prime}}^{\beta^{\prime} \alpha^{\prime}, \gamma \gamma^{\prime}} \\
& \times \frac{\theta\left(\tilde{E}_{s_{x} n_{x}}\right)-\theta\left(-\tilde{E}_{s_{x^{\prime}} n_{x^{\prime}}}\right)}{-\tilde{E}_{s_{x^{\prime}} n_{x^{\prime}}}-\tilde{E}_{s_{x} n_{x}}} .
\end{aligned}
$$

We can extract some physics from these formulas without calculating any numbers. Each interaction matrix element $\left(V_{x 4, x^{\prime} 1}^{\gamma \beta, \gamma^{\prime} \alpha}\right.$ from the ZS diagram, for example) comes with a factor of $e^{2} / \epsilon \ell_{0}$ according to Eq. (3). Therefore, once a factor of $\hbar \omega_{c}$ is pulled out of the denominator, the ZS, ZS', and BCS contributions to $u_{43 ; 21}^{(2)}$ are proportional to $\kappa\left(e^{2} / \epsilon \ell_{0}\right)$, as expected. In the lowest Landau level $(N=0)$, the ZS and ZS' terms vanish since there cannot be any hole excitations in the internal legs (the $x^{\prime} \gamma^{\prime}$ leg) since there are no filled Landau levels below in which to have virtual hole excitations. Therefore, only the BCS diagram renormalizes the two-body interactions in the lowest Landau level-incidentally, this is not the case for graphene since there all Landau levels $N=0$ are filled in which it is possible to excite virtual holes.

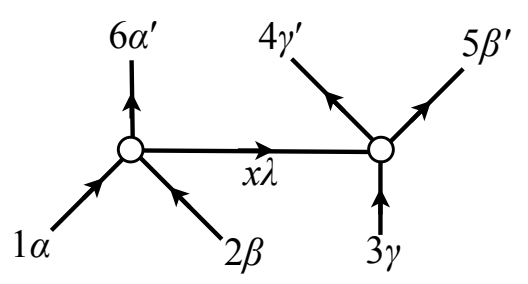

FIG. 3: One of the nine three-body diagrams.

We now turn to the three-body interaction $u_{654 ; 321}^{(3)}$. At order $\kappa$ there is a three-body term that is generated in our expansion of the action. Figure 3 shows one of the nine diagrams that contribute to the three-body term. The expression for the sum of all nine diagrams can be written compactly $\sqrt{59 / 76}$ as

$$
\sum_{n_{x}, s_{x}=0}^{\infty} \sum_{m_{x}=0}^{\infty} \sum_{\gamma=\uparrow, \downarrow} \sum_{\text {cyc. perm. }} \frac{V_{6 x, 21}^{\alpha^{\prime} \lambda, \beta \alpha} V_{54, x 3}^{\beta^{\prime} \gamma^{\prime}, \lambda \gamma}}{i \omega_{x}-\tilde{E}_{s_{x}, n_{x}}} .
$$

At low-frequencies, this leads to a three-body term:

$$
\begin{aligned}
u_{654: 321}^{(3)}= & -\sum_{n_{x}, s_{x}=0}^{\infty} \sum_{m_{x}=0}^{\infty} \sum_{\gamma=\uparrow, \downarrow} \\
& \times \sum_{\text {cyc. perm. }} \frac{V_{6 x, 21}^{\alpha^{\prime} \lambda, \beta \alpha} V_{54, x 3}^{\beta^{\prime} \gamma^{\prime}, \lambda \gamma}}{\tilde{E}_{s_{x}, n_{x}}}
\end{aligned}
$$

where we sum over all cyclic permutations of the indices labeled with $(1,2,3)$ and $(4,5,6)$. The prime on the summation over $n_{x}$ and $s_{x}$ indicates that we do not include cases where $s_{x}=0$ and $n_{x}=N$ simultaneously.

\section{B. Normal-Ordering the Three-Body Interaction}

There is one important subtlety that we now discuss. When we compute the three-body term, just discussed, that is induced at order $\kappa$, we evaluate diagrams that correspond to the operator expression:

$$
\frac{1}{2 !}[c_{6}^{\dagger} c_{x}^{\dagger} c_{2} c_{1} c_{5}^{\dagger} c_{4}^{\dagger} c_{x} c_{3}+c_{5}^{\dagger} c_{4}^{\dagger} c_{3} \overbrace{x} c_{x}^{\dagger} c_{6}^{\dagger} c_{2} c_{1}]
$$

Here, we have subsumed spin, Landau level, sub-band, and orbital indices into a single index for brevity. In the functional integral, we do not need to be careful about the order of Grassmann variables, apart from keeping track of signs. However, when we pass from the functional integral to the operator formalism, we must be careful about the order of these operators. The order of these operators is determined by a point-splitting regularization:

$$
\begin{aligned}
& \int d \omega e^{i \omega 0^{+}} c^{\dagger}(\omega) c(\omega)=c^{\dagger}(t) c(t) \\
& \int d \omega e^{i \omega 0^{-}} c^{\dagger}(\omega) c(\omega)=c(t) c^{\dagger}(t) .
\end{aligned}
$$

In other words, the order of operators at the same time is determined by whether the contour must be closed in the upper- or lower-half-plane. In the case of our threebody interaction, which is actually a retarded interaction, as in Eq. 200, this is determined by the sign of $\tilde{E}_{s_{x}, n_{x}}$. When we make the approximation of neglecting $\omega_{x}$ in the denominator of Eq. (20) to pass to the low-energy limit in Eq. 21, we can drop the $\omega_{x}$-dependence, except that we must remember the operator ordering that it imposes. Therefore, the three-body term that we have computed is not really

$$
-\sum \frac{V_{6 x, 21}^{\alpha^{\prime} \lambda, \beta \alpha} V_{54, x 3}^{\beta^{\prime} \gamma^{\prime}, \lambda \gamma}}{\tilde{E}_{s_{x}, n_{x}}} c_{6}^{\dagger} c_{5}^{\dagger} c_{4}^{\dagger} c_{3} c_{2} c_{1}
$$

but is, instead,

$$
\begin{aligned}
& -\frac{1}{2} \sum \frac{V_{6 x, 21}^{\alpha^{\prime} \lambda, \beta \alpha} V_{54, x 3}^{\beta^{\prime} \gamma^{\prime}, \lambda \gamma}}{\tilde{E}_{s_{x}, n_{x}}} \times \\
& {\left[\theta\left(\tilde{E}_{s_{x}, n_{x}}\right) c_{6}^{\dagger} c_{5}^{\dagger} c_{4}^{\dagger} c_{3} c_{2} c_{1}+\theta\left(-\tilde{E}_{s_{x}, n_{x}}\right) c_{6}^{\dagger} c_{2} c_{1} c_{5}^{\dagger} c_{4}^{\dagger} c_{3}\right.} \\
+ & \left.\theta\left(-\tilde{E}_{s_{x}, n_{x}}\right) c_{6}^{\dagger} c_{5}^{\dagger} c_{4}^{\dagger} c_{3} c_{2} c_{1}-\theta\left(\tilde{E}_{s_{x}, n_{x}}\right) c_{5}^{\dagger} c_{4}^{\dagger} c_{3} c_{6}^{\dagger} c_{2} c_{1}\right] .
\end{aligned}
$$


After we normal-order, this can be re-written as:

$$
\begin{aligned}
\frac{1}{3 !} u_{654 ; 321}^{(3)} c_{6}^{\dagger} c_{5}^{\dagger} c_{4}^{\dagger} c_{3} c_{2} c_{1} \\
+\sum \frac{V_{x 3, x_{0} 1}^{\gamma \alpha^{\prime}, \gamma^{\prime} \alpha} V_{4 x_{0}, 2 x}^{\beta^{\prime} \gamma^{\prime}, \beta \gamma}}{\tilde{E}_{s_{x}, n_{x}}} \theta\left(-\tilde{E}_{s_{x}, n_{x}}\right) c_{4}^{\dagger} c_{3}^{\dagger} c_{2} c_{1} \\
\quad-\sum \frac{V_{x 4, x_{0} 1}^{\gamma \beta^{\prime}, \gamma^{\prime} \alpha} V_{3 x_{0}, 2 x}^{\alpha^{\prime} \gamma^{\prime}, \beta \gamma}}{\tilde{E}_{s_{x}, n_{x}}} \theta\left(-\tilde{E}_{s_{x}, n_{x}}\right) c_{4}^{\dagger} c_{3}^{\dagger} c_{2} c_{1} \\
-\frac{1}{2} \sum \frac{V_{x x_{0}, 21}^{\gamma \gamma^{\prime}, \beta V_{43, x x_{0}}} V_{\beta^{\prime} \alpha^{\prime}, \gamma \gamma^{\prime}}}{\tilde{E}_{s_{x}, n_{x}}} \theta\left(\tilde{E}_{s_{x}, n_{x}}\right) c_{4}^{\dagger} c_{3}^{\dagger} c_{2} c_{1},
\end{aligned}
$$

where the $x_{0}$ indicates that $n_{x}=N$. Therefore, the renormalization of the two-body interaction takes the form

$$
\frac{1}{2 !}\left[\mathrm{ZS}+\mathrm{ZS}^{\prime}+\mathrm{BCS}\right] c_{4}^{\dagger} c_{3}^{\dagger} c_{2} c_{1}+\frac{1}{2 !} \delta \tilde{u}_{43 ; 21}^{(2)},
$$

where $\frac{1}{2 !} \delta \tilde{u}_{43 ; 21}^{(2)}$ is the sum of the last three terms in Eq. (26). Note that Eq. (26) contains the operators so when comparing the last three terms of Eq. (26) with Eqs. (17), 18), and (19), which do not contain the operators, there is a factor of 2 that multiplies the former compared to the latter. Also note that, in the $N=0$ Landau level, the first and second two-body terms in Eq. 26) vanish and only the third term contributes.

There is a simple check that shows that $\delta \tilde{u}_{43 ; 21}^{(2)}$ is necessary. Suppose that we compute two-particle scattering to second-order in the Coulomb interaction. We can do this either in the full theory (with all Landau levels kept) or in the low-energy effective theory of the $N^{\text {th }}$ Landau level. The answers must be the same either way. With the $\delta \tilde{u}_{43 ; 21}^{(2)}$ above, this is the case. There is a simple physical interpretation for the renormalization of the two-body term that results from normal-ordering the three-body term. We can think of this term as arising by taking a three-body vertex and connecting one of the incoming lines to one of the out-going lines in Fig. 3. If the two lines are connected to the same vertex (for example, 1 and 6 in Fig. 3) then we have a tadpole diagram, which vanishes. (Two such lines should not have been connected anyway since the operators at a single vertex are normal ordered.) If the two lines are connected to different vertices, then the resulting diagram is of the form of the ZS, ZS', or BCS diagrams (see Fig. 22), but one of the internal lines remains in the $N^{\text {th }}$ Landau level, rather than one of the higher or lower Landau levels that is being integrated out. In other words, this contribution accounts for processes such as the following: (i) two electrons in the $N^{\text {th }}$ Landau level interact, (ii) one of them is excited virtually to a higher Landau level while the other remains in the $N^{\text {th }}$ Landau level, and (iii) they interact again and the excited electron falls back to the $N^{\text {th }}$ Landau level.

One might worry that the result of such a process will depend on the couplings within the $N^{\text {th }}$ Landau level and its filling factor. The two-body terms that we have generated in this section derive from normal-ordering the three-body terms, so the dynamics within the $N^{\text {th }}$ Landau level does not enter into their computation. These dynamics (and the filling of the $N^{\text {th }}$ Landau level) enters only when we attempt to solve the resulting effective Hamiltonian for the $N^{\text {th }}$ Landau levels.

Another way to think about this is to recall that we have not integrated out states within the $N^{\text {th }}$ Landau level. Within a Wilsonian renormalization group scheme, which is essentially what we have adopted, all internal lines in diagrams are at high energies and all external lines are at low energies because we integrate out $N^{\prime} \neq N$ Landau levels but do not do anything to states in the $N^{\text {th }}$ Landau level, which will be dealt with later (by, for instance, exact diagonalization). In our case, this means that all internal lines are in the $N^{\prime} \neq N$ Landau levels while all external lines are in the $N^{\text {th }}$ Landau level. In such an approach, a diagram with $k$ external legs in which $p$ internal lines are in the $N^{\prime} \neq N$ Landau levels while $q$ are in the $N^{\text {th }}$ Landau level arises in the following way. When we integrate out the $N^{\prime} \neq N$ Landau levels, we will generate a vertex with $k+2 q$ legs. One of the terms contributing to this vertex will have $p$ internal lines. If we were to solve the problem in the $N^{\text {th }}$ Landau level perturbatively, then there would be a diagram in which we took this $(k+2 q)$-leg vertex and connected $q$ incoming lines to $q$ outgoing lines. Therefore, this physics is present in the $(k+2 q)$-leg vertex. In our case, this means that two-body processes in which the intermediate state has one electron in the $N^{\text {th }}$ Landau level and one in an $N^{\prime} \neq$ $N$ Landau level are present in our three-body vertex. However, to correctly account for them, it is crucial to order the operators in the three-body vertex correctly.

Note that in Fermi liquid theory for electrons with no external magnetic field $(B=0)$ we never have to consider the type of virtual process which gives this contribution because momentum conservation does not allow processes in which one electron is scattered to momenta far from the Fermi surface while the other electron stays near the Fermi surface. In low Landau levels, by contrast, there is no conservation law precluding such processes and they are not only present but, in fact, give a substantial contribution to the renormalization of the two-body interaction.

\section{Effective Hamiltonian}

Once we have these new interactions, $u_{43 ; 21}^{(2)}$ and $u_{654 ; 321}^{(3)}$, we can calculate the Haldane pseudopotentials and their three-body generalizations ${ }^{77}$. We use $V_{M S}^{(2)}(N)$ to denote the bare two-body pseudopotential for two electrons residing in the $N^{\text {th }}$ Landau level with relative angular momentum $M$ and total spin $S$. The $\mathcal{O}(\kappa)$ correction (including the contribution discussed in the previous subsection) to this pseudopotential is denoted by $\delta V_{M S}^{(2)}(N)$. The three-body pseudopotential for three electrons with relative angular momentum $M$ (defined as the degree of 
the relative wavefunction $)^{77}$ and total spin $S$ is denoted by $V_{M S}^{(3)}(N)$ :

$$
\begin{array}{r}
V_{M S}^{(2)}(N)=\sum\left\langle M S \mid m_{3} \alpha^{\prime}, m_{4} \beta^{\prime}\right\rangle\left\langle m_{1} \alpha, m_{2} \beta \mid M S\right\rangle \\
\times V_{43 ; 21}
\end{array}
$$

$$
\begin{array}{r}
\delta V_{M S}^{(2)}(N)=\sum\left\langle M S \mid m_{3} \alpha^{\prime}, m_{4} \beta^{\prime}\right\rangle\left\langle m_{1} \alpha, m_{2} \beta \mid M S\right\rangle \\
\times \delta u_{43 ; 21}^{(2)}
\end{array}
$$

$$
\begin{aligned}
V_{M S}^{(3)}(N)=\sum\left\langle M S \mid m_{4} \gamma^{\prime}, m_{5} \beta^{\prime}, m_{6} \alpha^{\prime}\right\rangle \times \\
\left\langle m_{1} \alpha, m_{2} \beta, m_{3} \gamma \mid M S\right\rangle u_{654 ; 321}^{(3)}
\end{aligned}
$$

where $\sum$ indicates a sum over all $m_{i}$ and primed spin variables.

We will drop the spin indices from the pseudopotentials we are considering in this work and write $V_{M}^{(2)}(N) \equiv$ $V_{M S}^{(2)}(N), V_{M}^{(3)}(N) \equiv V_{M S}^{(3)}(N)$ where the spin will be $S=$ $1 / 2$ for $M=1$ and 2 and $S=3 / 2$ for $M \geq 3$ for the threebody case, while for the two-body case, the spin will be $S=1(0)$ for spin polarized (unpolarized) electrons, i.e., $M=$ odd (even). Our Hamiltonian is appropriate for all filling factors. Therefore, we calculate even and odd $M$ for the two-body terms in addition to $M=1,2,3,5,6,7$, and 8 for the three-body terms. It should be clear what the total spin is for those particular $M$ 's. As shown in Section III the numerical value for $V_{8}^{(3)}(0), V_{8}^{(3)}(1)$ are very small, so we ignore $M>8$. This allows us to avoid the potential complication of pseudopotential matrices, instead of pseudopotential numbers, that arise for total relative angular momentum $M \geq 9$ due to the multiple three-particle wavefunctions 57 .

Starting with Eq. (2), we have chosen to calculate the effective interactions and the resulting pseudopotentials in the planar geometry, rather than in the spherical geometry. For the two-body pseudopotentials, we are only reporting the corrections due to Landau level and subband mixing and one must consider these corrections as corrections to the planar bare pseudopotentials. In order to calculate them in the spherical geometry, we would have to recalculate the corrections for each system size, which is extremely cumbersome and, presumably unnecessary (when we extrapolate to the thermodynamic limit). The spherical pseudopotentials approach the planar pseudopotentials in the thermodynamic limit of a large sphere and it has been argued ${ }^{34 \mid 35}$ that using the planar pseudopotentials in finite systems better approximate the infinite system.

With these new effective pseudopotentials, we construct a Hamiltonian for the FQHE in the $N^{\text {th }}$ Landau level which includes Landau level and sub-band mixing effects perturbatively to first order in $\kappa$,

$$
\begin{aligned}
\hat{H}_{\text {eff }}\left(\kappa, d / \ell_{0}\right)=\hat{V}_{0}^{(2)}\left(d / \ell_{0}\right)+\kappa \hat{V}_{1}^{(2)} & \left(d / \ell_{0}\right) \\
& +\kappa \hat{V}_{1}^{(3)}\left(d / \ell_{0}\right)
\end{aligned}
$$

where

$$
\begin{array}{rl}
\hat{V}_{0}^{(2)}\left(d / \ell_{0}\right)=\sum_{i<j} & V\left(\left|\mathbf{r}_{i}-\mathbf{r}_{j}\right|\right) \\
& =\sum_{M} V_{M}^{(2)}\left(N, d / \ell_{0}\right) \sum_{i<j} \hat{P}_{i j}(M)
\end{array}
$$

is the bare pseudopotentials with $V\left(\left|\mathbf{r}_{i}-\mathbf{r}_{j}\right|\right)$ being the usual finite-thickness augmented Coulomb interaction (see Refs. 34 35); it is a function of the quantum-well thickness $d / \ell_{0}$. In this expression, $\hat{P}_{i j}(M)$ is an operator that projects the pair of electrons $(i, j)$ onto a two-body state of relative angular momentum $M$. Similarly,

$$
\hat{V}_{1}^{(2)}\left(d / \ell_{0}\right)=\sum_{M} \delta V_{M}^{(2)}\left(N, d / \ell_{0}\right) \sum_{i<j} \hat{P}_{i j}(M)
$$

and

$$
\hat{V}_{1}^{(3)}\left(d / \ell_{0}\right)=\sum_{L} V_{M}^{(3)}\left(N, d / \ell_{0}\right) \sum_{i<j<k} \hat{P}_{i j k}(M)
$$

where $\hat{P}_{i j k}(M)$ is a projection operator that projects onto triplets of electrons with relative angular momentum $M$. The subscripts 0 and 1 in Eq. (31) indicate the bare interaction and the first order corrections, respectively. We also briefly comment about the projection operators: what is often not explicitly written is that the $m$-body projection operators $\hat{P}_{i j \ldots m}(M)$ have normalization set by the property that their eigenvalues must be 0 or 1 .

Since every Landau level has the same number of states, we project the $N^{\text {th }}$ Landau level to the $N=0$ Landau level. The difference between the $N^{\text {th }}$ Landau level and the zeroth is reflected in the values of the pseudopotentials. However, from the point of view of any calculations, our effective Hamiltonian, Eq. (31), is a Hamiltonian for electrons in the lowest Landau level that simulates the Hamiltonian for electrons in the $N^{\text {th }}$ Landau level.

\section{THE EFFECTIVE TWO- AND THREE-BODY PSEUDOPOTENTIALS FOR GaAs}

We now discuss the numerical values of the two- and three-body pseudopotentials in the presence of Landaulevel and sub-band mixing appropriate to GaAs (i.e. for parabolic $B=0$ bands). For the two-body case, we calculate Eq. (27). The result is a function of singleparticle angular momenta $m_{1}, m_{2}, m_{3}$, and $m_{4}$, denoted by $\delta u_{43: 21}^{(2)}$, which depends on the Landau level index $N$ of the partially-filled Landau level which we retain. All other Landau levels are either completely empty or completely full and are integrated out. Then, $\delta u_{43: 21}^{(2)}$ is substituted into Eq. 29, yielding the two-body pseudopotential correction $\delta V_{M}^{(2)}(N)$. For the three-body term we calculate $u_{654: 321}^{(3)}$ (Eq. 20p) which is a function of angular momenta $m_{6}, m_{5}, m_{4}, m_{3}, m_{2}$, and $m_{1}$. The result, 
which depends on the index $N$ of the partially-filled Landau level which we retain, is substituted into Eq. (30), yielding the three-body pseudopotential $V_{M}^{(3)}(N)$.

However, Eqs. (27), and (20) all contain infinite sums over Landau levels and sub-bands and none of these sums can be done analytically. Therefore, they must be truncated and the resulting finite sums must be computed numerically. The infinite sum is the limit of the finite sums as the truncation is taken to infinity. We calculate our final results $\left[\delta V_{M}^{(2)}\left(N, d / \ell_{0}\right)\right.$ and $\left.V_{M}^{(3)}\left(N, d / \ell_{0}\right)\right]$ as a function of the truncation and determine the convergence to the infinite sum by fitting to some chosen general common function.

TABLE I: We list the numerical values of $\delta V_{M}^{(2)}\left(N, d / \ell_{0}\right)$ for $d / \ell_{0}=0,1,2,3,4$ and $N=0,1$. Values for $d / \ell_{0}=0$ were given previously in Ref. 59 for $M=1,3$ but due to the new two-body correction coming from properly normal-ordering the three-body interaction the values have been changed $\sqrt{78}$. All energies are given in units of $e^{2} / \epsilon \ell_{0}$.

\begin{tabular}{lrrrrr}
\hline \hline & $d / \ell_{0}=0$ & 1 & 2 & 3 & 4 \\
\hline$\delta V_{0}^{(2)}\left(0, d / \ell_{0}\right)$ & -0.3422 & -0.1963 & -0.1281 & -0.0900 & -0.0665 \\
$\delta V_{1}^{(2)}\left(0, d / \ell_{0}\right)$ & -0.0328 & -0.0300 & -0.0254 & -0.0211 & -0.0175 \\
$\delta V_{2}^{(2)}\left(0, d / \ell_{0}\right)$ & -0.0112 & -0.0108 & -0.0098 & -0.0088 & -0.0077 \\
$\delta V_{3}^{(2)}\left(0, d / \ell_{0}\right)$ & -0.0055 & -0.0054 & -0.0051 & -0.0047 & -0.0043 \\
$\delta V_{4}^{(2)}\left(0, d / \ell_{0}\right)$ & -0.0033 & -0.0032 & -0.0031 & -0.0029 & -0.0027 \\
$\delta V_{5}^{(2)}\left(0, d / \ell_{0}\right)$ & -0.0022 & -0.0022 & -0.0021 & -0.0020 & -0.0019 \\
$\delta V_{6}^{(2)}\left(0, d / \ell_{0}\right)$ & -0.0016 & -0.0015 & -0.0015 & -0.0014 & -0.0014 \\
$\delta V_{7}^{(2)}\left(0, d / \ell_{0}\right)$ & -0.0012 & -0.0011 & -0.0011 & -0.0011 & -0.0010 \\
$\delta V_{8}^{(2)}\left(0, d / \ell_{0}\right)$ & -0.0009 & -0.0009 & -0.0009 & -0.0009 & -0.0008 \\
$\delta V_{9}^{(2)}\left(0, d / \ell_{0}\right)$ & -0.0007 & -0.0007 & -0.0007 & -0.0007 & -0.0007 \\
\hline$\delta V_{0}^{(2)}\left(1, d / \ell_{0}\right)$ & -0.3816 & -0.3184 & -0.2696 & -0.2307 & -0.1997 \\
$\delta V_{1}^{(2)}\left(1, d / \ell_{0}\right)$ & -0.2143 & -0.2020 & -0.1815 & -0.1617 & -0.1442 \\
$\delta V_{2}^{(2)}\left(1, d / \ell_{0}\right)$ & -0.1787 & -0.1456 & -0.1296 & -0.1172 & -0.1067 \\
$\delta V_{3}^{(2)}\left(1, d / \ell_{0}\right)$ & -0.1039 & -0.0986 & -0.0927 & -0.0868 & -0.0812 \\
$\delta V_{4}^{(2)}\left(1, d / \ell_{0}\right)$ & -0.0789 & -0.0729 & -0.0694 & -0.0664 & -0.0635 \\
$\delta V_{5}^{(2)}\left(1, d / \ell_{0}\right)$ & -0.0353 & -0.0423 & -0.0458 & -0.0473 & -0.0476 \\
$\delta V_{6}^{(2)}\left(1, d / \ell_{0}\right)$ & -0.0258 & -0.0296 & -0.0334 & -0.0361 & -0.0376 \\
$\delta V_{7}^{(2)}\left(1, d / \ell_{0}\right)$ & -0.0115 & -0.0181 & -0.0234 & -0.0272 & -0.0297 \\
$\delta V_{8}^{(2)}\left(1, d / \ell_{0}\right)$ & -0.0073 & -0.0123 & -0.0175 & -0.0216 & -0.0245 \\
$\delta V_{9}^{(2)}\left(1, d / \ell_{0}\right)$ & -0.0023 & -0.0079 & -0.0133 & -0.0176 & -0.0209 \\
\hline \hline & & & & & \\
\hline
\end{tabular}

The two-body pseudopotential corrections and threebody pseudopotentials are given in, respectively, Tables II and III for $d / \ell_{0}=0,1,2,3$, and 4 and shown graphically in Fig. 4 up to $d / \ell_{0}=8$. The most salient feature is that these corrections are small compared to the bare pseudopotentials in the $N=0$ Landau level (except for $M=0$ ) and the $N=1$ Landau level (except for $M \leq 3$ ). This was already noted in Ref. 59 for $d / \ell_{0}=0$, however, without taking into account the modified two-body contribution. (Note that the numerical values which we find for $d / \ell_{0}=0$ are different than in Ref. 59 due primarily to the correction to the two-body term coming from
TABLE II: We list the numerical values of the three-body pseudopotentials $V_{M}^{(3)}\left(N, d / \ell_{0}\right)$ as a function of $M$ for $d / \ell_{0}=$ $0,1,2,3$, and 4 and $N=0,1$. The values for $d / \ell_{0}=0$ were given previously in Ref. $[59$ where again a few values have been changed very slightly due to a more careful extrapolation. All energies are given in units of $e^{2} / \epsilon \ell_{0}$.

\begin{tabular}{lrrrrr}
\hline \hline & $d / \ell_{0}=0$ & 1 & 2 & 3 & 4 \\
\hline$V_{1}^{(3)}\left(0, d / \ell_{0}\right)$ & -0.0345 & -0.0400 & -0.0364 & -0.0312 & -0.0263 \\
$V_{2}^{(3)}\left(0, d / \ell_{0}\right)$ & -0.0540 & -0.0410 & -0.0324 & -0.0262 & -0.0216 \\
$V_{3}^{(3)}\left(0, d / \ell_{0}\right)$ & -0.0181 & -0.0173 & -0.0156 & -0.0138 & -0.0120 \\
$V_{5}^{(3)}\left(0, d / \ell_{0}\right)$ & 0.0033 & 0.0026 & 0.0015 & 0.0006 & -0.0000 \\
$V_{6}^{(3)}\left(0, d / \ell_{0}\right)$ & -0.0107 & -0.0102 & -0.0093 & -0.0083 & -0.0073 \\
$V_{7}^{(3)}\left(0, d / \ell_{0}\right)$ & 0.0059 & 0.0054 & 0.0043 & 0.0033 & 0.0025 \\
$V_{8}^{(3)}\left(0, d / \ell_{0}\right)$ & -0.0047 & -0.0045 & -0.0041 & -0.0037 & -0.0033 \\
\hline$V_{1}^{(3)}\left(1, d / \ell_{0}\right)$ & -0.0319 & -0.0280 & -0.0232 & -0.0192 & -0.0160 \\
$V_{2}^{(3)}\left(1, d / \ell_{0}\right)$ & -0.0305 & -0.0223 & -0.0174 & -0.0142 & -0.0117 \\
$V_{3}^{(3)}\left(1, d / \ell_{0}\right)$ & -0.0147 & -0.0136 & -0.0118 & -0.0101 & -0.0087 \\
$V_{5}^{(3)}\left(1, d / \ell_{0}\right)$ & -0.0054 & -0.0051 & -0.0047 & -0.0042 & -0.0038 \\
$V_{6}^{(3)}\left(1, d / \ell_{0}\right)$ & -0.0099 & -0.0093 & -0.0082 & -0.0071 & -0.0061 \\
$V_{7}^{(3)}\left(1, d / \ell_{0}\right)$ & 0.0005 & 0.0002 & -0.0001 & -0.0004 & -0.0006 \\
$V_{8}^{(3)}\left(1, d / \ell_{0}\right)$ & -0.0009 & -0.0013 & -0.0016 & -0.0017 & -0.0018 \\
\hline \hline
\end{tabular}

the correct normal-ordering of the three-body term.) By "small compared to the bare pseudopotential", we mean that the coefficients (for $M>0$ in the $N=0$ Landau level and $M \leq 3$ for the $N=1$ Landau level) are $\approx 10$ times smaller and for increasing angular momentum $M$ approximately 50 times smaller. The smallness of these coefficients means that it is conceivable that our perturbative calculation is valid even for $\kappa$ beyond one. However, in order to say this with certainty, we would need to compute the order $\kappa^{2}, \kappa^{3}, \ldots$ terms and show that the series converges.

From Fig. 4, we can see the dependence of the twobody corrections to the bare pseudopotentials on $d / \ell_{0}$. We observe in both the lowest and first excited Landau levels that generally the magnitude of the corrections to the two-body pseudopotentials decrease with increasing $d / \ell_{0}$; remember that these numbers will be multiplied by $\kappa$ when constructing the full two-body pseudopotentials. The bare Coulomb interaction is itself also softened as $d / \ell_{0}$ increases and the fractional change in the bare pseudopotential and its two-body correction is similar. This behavior begins to break down very slightly in the $N=1$ Landau level at larger values of $M$ where some mild nonmonotonic behavior is observed, however, we emphasize that the relative value of the correction is quite small for higher $M$ 's and this non-monotonic behavior will most likely have little effect on the physics. The two-body corrections decrease with increasing $d / \ell_{0}$ and, eventually are expected to vanish, so in essence, the effect of finitethickness is to mitigate the effect of Landau level mixing.

In Fig. 5 we show the two-body corrections to the bare pseudopotentials as a function of relative angular mo- 

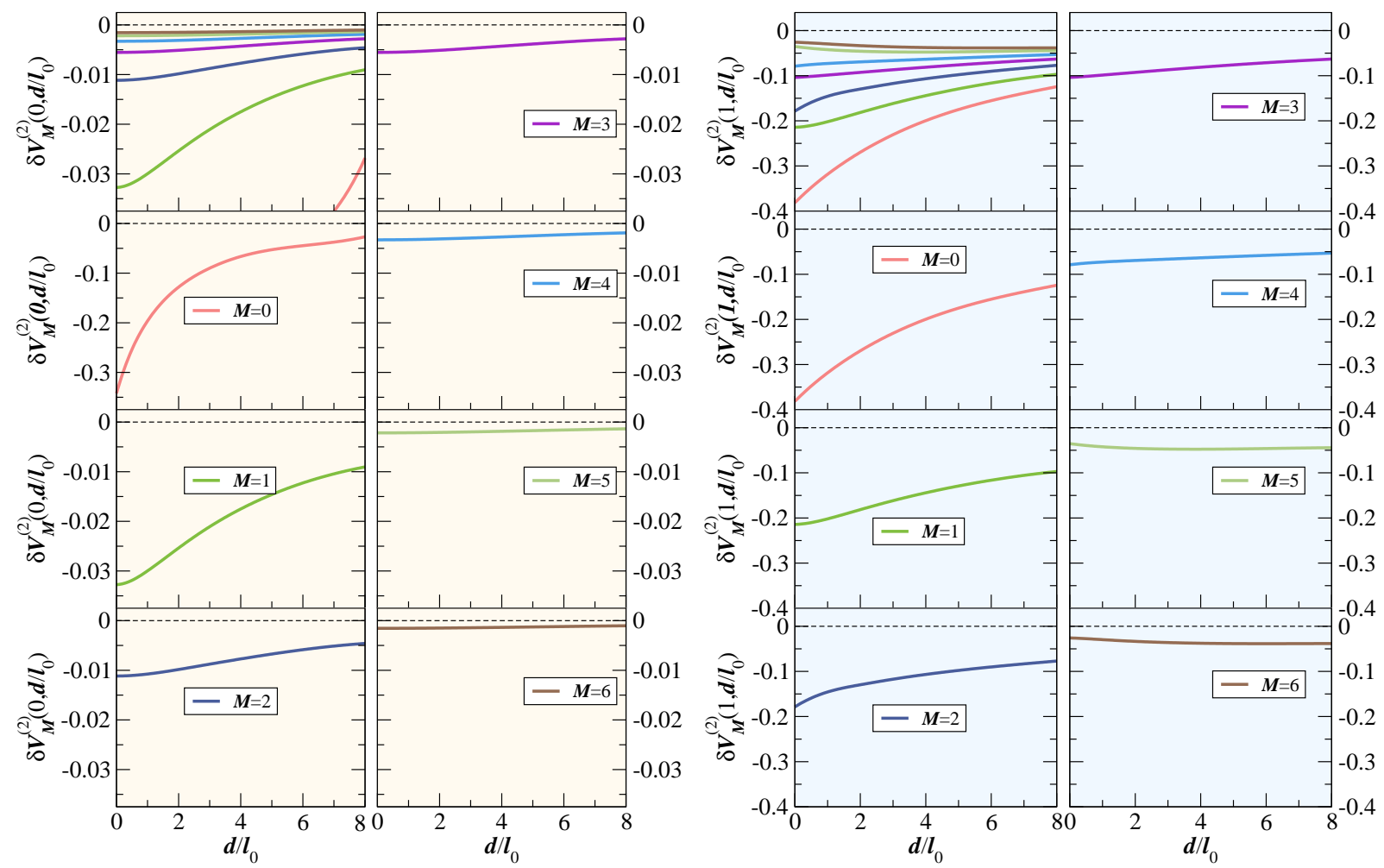

FIG. 4: (Color online) The Landau level mixing induced corrections to the two-body pseudopotentials for $M=1-6$ in the lowest $\left(N=0\right.$, left figures) and second $\left(N=1\right.$, right figures) Landau levels. The top left plot in both panels shows all $\delta V_{M}^{(2)}\left(N, d / \ell_{0}\right)$ versus $d / \ell_{0}$ on the same plot for an easier comparison. Note that for the lowest Landau level $(N=0)$ the $M=0$ plot is on a different vertical scale than all the others since its value is so much larger. For the $N=0$ Landau level, the corrections are less than $10 \%$ (for all $d / \ell_{0}$ ) of the bare pseudopotential value-discounting $M=0$ which is nearly $30 \%$ (for $d / \ell_{0}=0$ ) the bare pseudopotential. For the $N=1$ the corrections are much larger. For $M=0$ the correction is nearly $60 \%$ and remains near $50 \%$ for $M<3$. Of course, for both Landau levels the corrections are mitigated by finite width (increasing $\left.d / \ell_{0}\right)$. All energies are given in units of $e^{2} / \epsilon \ell_{0}$.

mentum $M$ for $d / \ell_{0}=0,1,2,3$, and 4 for $N=0$ and $N=1$. As in the case of the bare pseudopotentials we observe that the two-body corrections decrease with both increasing $M$ and $d / \ell_{0}$. However, an effect not seen in the bare pseudopotentials for $N=1$ is observed in the two-body corrections; they are larger in magnitude than their $N=0$ counterparts.

Since there is no three-body term at tree-level, the $\mathcal{O}(\kappa)$ correction is the leading three-body term. Although the three-body interaction is numerically small, as may be seen in Table II and Figs. 6 and 7, it is the leading term which breaks particle-hole symmetry. The three-body term is especially interesting for the FQHE at $\nu=5 / 2$. In its absence, particle-hole symmetry is a symmetry of the Hamiltonian and, consequently, the most promising candidates to describe the the $5 / 2$ FQHE, the Moore-Read Pfaffian state and the anti-Pfaffian state, would be exactly degenerate. As is well known ${ }^{79180}$, the Moore-Read Pfaffian is the exact zero-energy solution to a repulsive (positive) three-body Hamiltonian that only contains an $M=3$ term. The anti-Pfaffian, of course, is the exact zero-energy solution to the Hamiltonian that is the particle-hole conjugate of the one that yields the Moore-Read Pfaffian. Under particle-hole conjugation, the positive $M=3$ three-body term picks up a minus sign and becomes attractive. Additionally, however, a two-body term is also generated under particle-hole conjugation of the Moore-Read Pfaffian Hamiltonian. The $M=3$ three-body pseudopotential due to Landau level mixing is negative in both the $N=0$ and $N=1$ Landau levels, a sign which, naively, favors the anti-Pfaffian state over the Moore-Read Pfaffian. However, with the $M=5$, 6,7 , and 8 three-body terms being non-zero, one cannot predict the physics from the pseudopotentials alone.

To illustrate the $d / \ell_{0}$ dependence of the three-body pseudopotentials, we show $V_{M}^{(3)}\left(N, d / \ell_{0}\right)$ as a function of $d / \ell_{0}$ and $M$ in, respectively, the $N=0$ and $N=$ 1 Landau levels in Figs. 6 and 7. Other than the $M=1, N=0$ case (discussed below), finite width decreases $V_{M}^{(3)}\left(N, d / \ell_{0}\right)$ monotonically. Figure 7 shows $V_{M}^{(3)}\left(N, d / \ell_{0}\right)$ as a function of $M$ and we see that for 


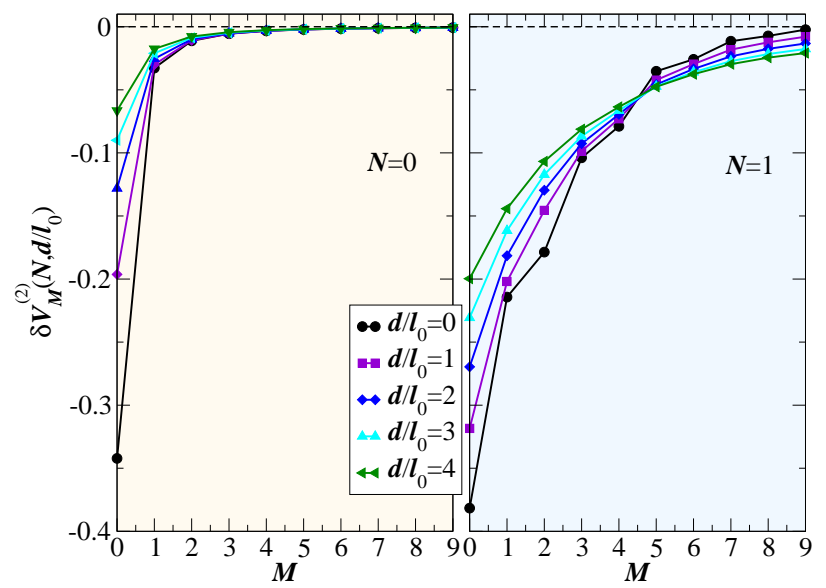

FIG. 5: (Color online) $\delta V_{M}^{(2)}\left(N, d / \ell_{0}\right)$ as a function of $M$ for $d / \ell_{0}=0,1,2,3$ and 4 . The left panel corresponds to the lowest Landau level $(N=0)$ while the right panel corresponds to the first excited Landau level $(N=1)$. As in the case of the bare pseudopotentials, the corrections to the two-body pseudopotentials decrease with increasing $M$. All energies are given in units of $e^{2} / \epsilon \ell_{0}$.

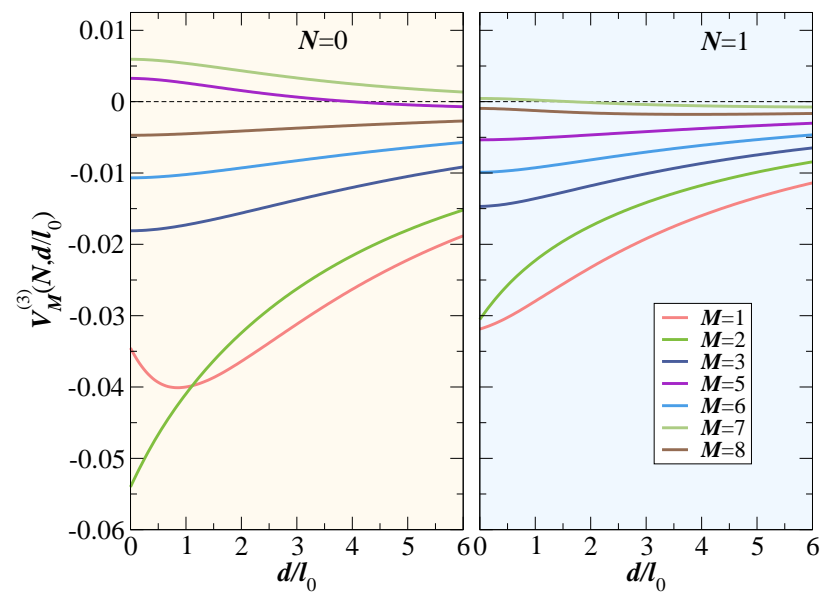

FIG. 6: (Color online) The three-body pseudopotentials $V_{M}^{(3)}\left(N, d / \ell_{0}\right)$ as a function of $d / \ell_{0}$ for the lowest Landau level (left panel) and the first excited Landau level (right panel). Note that these are numerically small compared to the bare two-body pseudopotentials. All energies are given in units of $e^{2} / \epsilon \ell_{0}$.

$M \geq 5$ the absolute values oscillate: $\left|V_{5}^{(3)}\left(N, d / \ell_{0}\right)\right|<$ $\left|V_{6}^{(3)}\left(N, d / \ell_{0}\right)\right|,\left|V_{7}^{(3)}\left(N, d / \ell_{0}\right)\right|<\left|V_{6}^{(3)}\left(N, d / \ell_{0}\right)\right|$, etc.

We note that there is some non-trivial behavior in the $M=1$ and 2 three-body pseudopotentials for small values of $d / \ell_{0}$ for $N=0,1$. The $M=1$ pseudopotential in the lowest Landau level is non-monotonic and starts
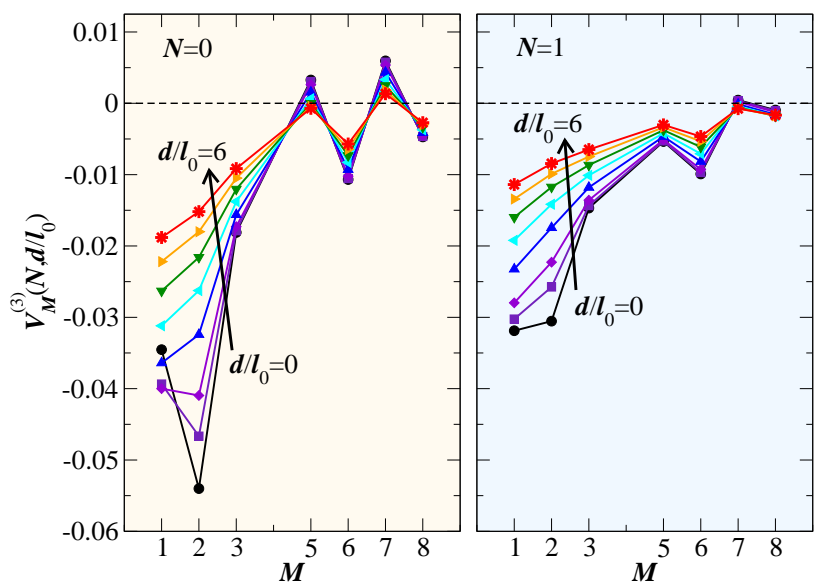

FIG. 7: (Color online) $V_{M}^{(3)}\left(N, d / \ell_{0}\right)$ as a function of $M$ for $d / \ell_{0}=0,0.5,1-6$ for the lowest Landau level (left panel) and the first excited Landau level (right panel). The magnitude decreases with $M$ except for the non-trivial oscillatory behavior for $M \geq 5$. All energies are given in units of $e^{2} / \epsilon \ell_{0}$.

out smaller than the $M=2$ pseudopotential. As $d / \ell_{0}$ increases, the two cross and $M=1$ is larger than $M=2$ for large $d / \ell_{0}$. In the $N=1$ Landau level, we see the same qualitative behavior except that $M=1$ and 2 do not change signs.

Finally, we examine the overall structure of the effective interaction by showing $V_{M}^{(p)}\left(N, d / \ell_{0}\right)$ for $N=0,1$ (top and bottom panels, respectively) and $p=2,3$ in Fig. 8 as a function of $M$ for a range of $\kappa$. The two- (three-) body pseudopotentials are color-coded with "hot" ("cold") colors going from red to yellow (green to blue) as $\kappa$ is changed from 0 to 3 . The numerical values of the corrections to the bare two-body pseudopotentials and the three-body pseudopotentials are also small compared to the bare two-body pseudopotentials. However, as noted in Sec. I. small quantitative changes to the pseudopotentials can have large qualitative effects on the possible ground states of the effective FQHE Hamiltonian. As noted above, naively one might expect that the negative value of the $M=3$ three-body term might favor the formation of the anti-Pfaffian, but for values of $\kappa>1$ there is a fair amount of renormalization of the two-body pseudopotentials. It is known from previous work ${ }^{34 / 35}$ that finite thickness stabilizes the Moore-Read Pfaffian at $\nu=5 / 2$, but this calculation was only done for $\kappa=0$ and could not distinguish between the Moore-Read Pfaffian and anti-Pfaffian. From examining the Landau level and sub-band mixing induced corrections to the Haldane pseudopotentials, there is potentially a range of $d / \ell_{0}$ and $\kappa$ that would favor or disfavor the Moore-Read Pfaffian and anti-Pfaffian. In fact, a cursory look at the two-body pseudopotentials leads one to suspect that Landau level mixing is relatively unimportant for spin-polarized elec- 


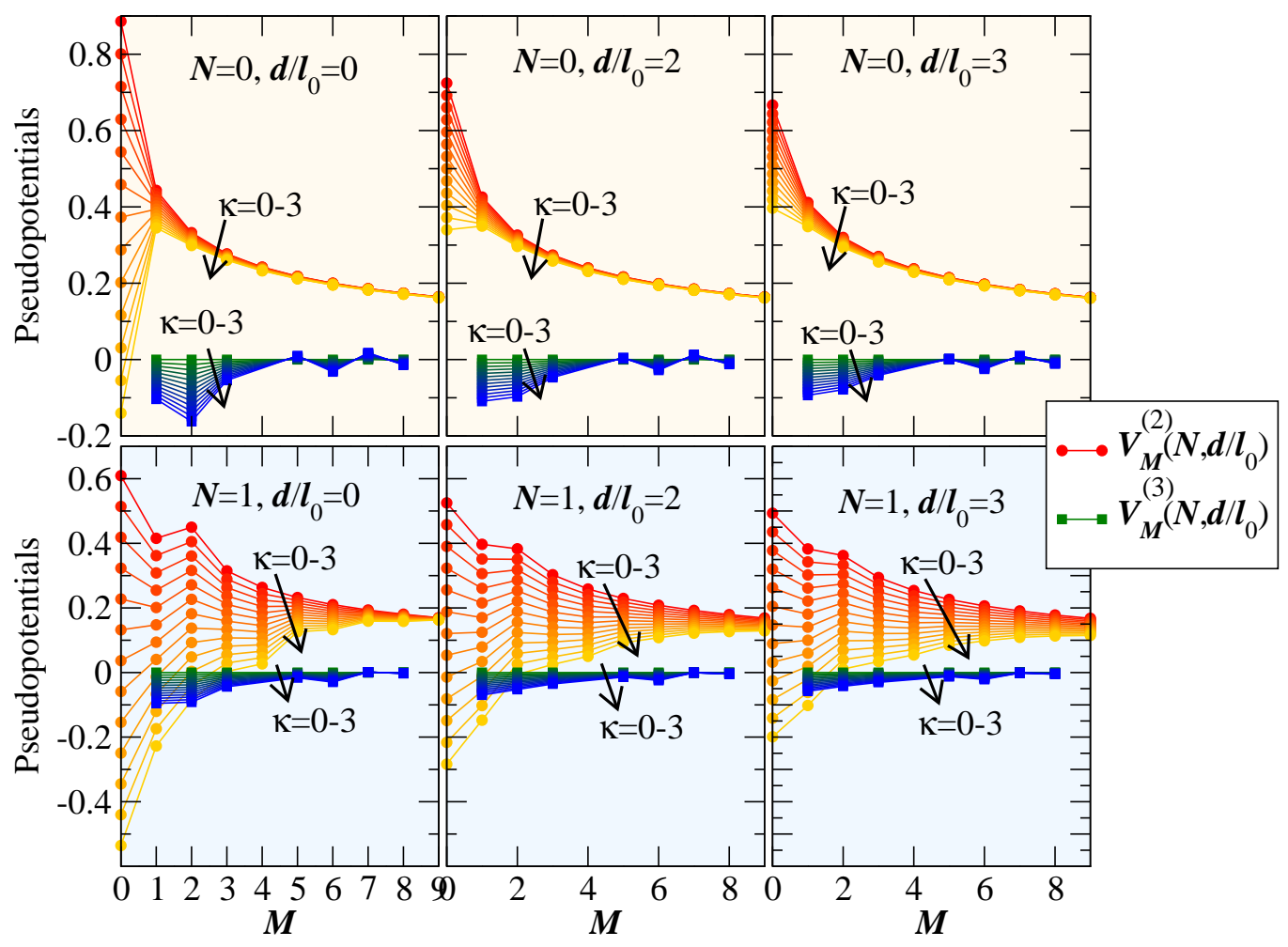

FIG. 8: (Color online) The two-body and three-body pseudopotentials, $V_{M}^{(2)}\left(N, d / \ell_{0}\right)$ and $V_{M}^{(3)}\left(N, d / \ell_{0}\right)$, as functions of $M$ for $\kappa=0-3$ for $N=0$ (top panels), $N=1$ (bottom panels) for $d / \ell_{0}=0$ (left), 2(middle), and 3(right). Obviously, $V_{M}^{(2)}\left(N, d / \ell_{0}\right)$ is a sum of the bare two-body term plus $\kappa$ times the two two-body corrections. The two-body pseudopotentials are color-coded such that $\kappa=0$ corresponds to red and $\kappa=3$ corresponds to yellow, i.e., the color-coding is "hot". The three-body terms are color-coded "cold", i.e., $\kappa=0$ is green and the color changes continuously to blue for $\kappa=3$. Generally, increasing $d / \ell_{0}$ and $\kappa$ reduces the two-body pseudopotentials compared to the bare values $(\kappa=0)$. The three-body pseudopotentials are directly proportional to $\kappa$ so increase in magnitude for increasing $\kappa$, however, increasing $d / \ell_{0}$ mitigates this effect by decreasing the coefficients themselves, as expected. Please note that any two-body corrections for $M \geq 10$ are expected to be small and unimportant. From inspection of this figure one would expect that Landau level mixing is likely to be unimportant for the FQHE in the $N=0$ Landau level for spin-polarized electrons. For the $N=1$ Landau level, however, it is not clear what effect these changes to the pseudopotentials will have on the physics and the answer will have to await future exact diagonalization studies. Of course, even in the $N=0$ Landau level the effect of the three-body terms cold produce non-trivial effects. All energies are given in units of $e^{2} / \epsilon \ell_{0}$.

trons in the $N=0$ Landau level, while, evidently, Landau level mixing is much more important in the $N=1$ Landau level. However, we emphasize that the effect of the three-body terms on the physics of the FQHE in either the $N=0$ or 1 Landau levels is non-trivial and not known. We are currently undertaking a thorough numerical study to determine the full quantum phase diagram of this new Hamiltonian for GaAs 81 .

We now turn our attention to the study of an effective Hamiltonian for graphene.

\section{EFFECTIVE HAMILTONIAN FOR GRAPHENE}

The formalism for including Landau level mixing in graphene (linear dispersion at $B=0$ ) is similar to that of GaAs, so we only highlight the differences, such as the absence of finite thickness. The Landau level problem has been investigated by many authors $\sqrt{53} \sqrt{58}$ and we only briefly repeat those results that are relevant to the present discussion.

The action [Eq. (1)] for graphene is of the same form as for GaAs with three differences. The first, and most important, is that the kinetic energy of an electron in the $N^{\text {th }}$ Landau level is $E_{n}^{\text {graph }}=\operatorname{sgn}(n) \sqrt{2|n|} \hbar v_{F} / \ell_{0}$ where $v_{F}$ is the electron Fermi velocity $\left(\sim 10^{6} \mathrm{~m} / \mathrm{s}\right)$. Unlike 
in GaAs, this kinetic energy can be positive or negative (electrons or holes) and increases as the square root of the absolute value of the Landau level index $n$. Hence, the Landau levels in graphene are not equally spaced; the spacing between successive Landau levels decreases (as the inverse square root) as the Landau level index is increased. The second important difference is that there is no sub-band index " $s$ " since the thickness of a graphene monolayer is atomically thin. The third and final difference lies in the interaction matrix elements. We will use the notation $V_{43,21}^{\text {graph }}$ for the matrix elements of the Coulomb interaction for electrons in graphene and we will continue to use $V_{43,21}$ for the corresponding matrix elements in GaAs.

In GaAs, the electron-electron interaction matrix element $V_{43,21}$ is given in Eq. (3). In graphene, Eq. (3) is modified as

$$
\begin{aligned}
V_{43,21}^{\text {graph }}= & \int \frac{d^{2} k}{2 \pi} V(k) e^{-k^{2}} \frac{\sqrt{2}^{\sum_{i=1}^{4} \delta_{n_{i}, 0}}}{4} G_{m_{4} m_{2}}(-k) G_{m_{3} m_{1}}(k) \\
& \times\left\{\operatorname{sgn}\left(n_{4}\right) \operatorname{sgn}\left(n_{2}\right) G_{\left|n_{4}\right|-1,\left|n_{2}\right|-1}(-\bar{k})+G_{\left|n_{4}\right|\left|n_{2}\right|}(-\bar{k})\right\} \\
& \times\left\{\operatorname{sgn}\left(n_{3}\right) \operatorname{sgn}\left(n_{1}\right) G_{\left|n_{3}\right|-1,\left|n_{1}\right|-1}(\bar{k})+G_{\left|n_{3}\right|\left|n_{1}\right|}(\bar{k})\right\}
\end{aligned}
$$

where we set $\operatorname{sgn}(0)=0$ in the above equation. In this equation, $V(k)=e^{2} /\left(2 \pi \epsilon \ell_{0} k\right)$ is the pure (i.e. zero-thickness) Coulomb interaction. Note that the definition of $V_{43,21}^{\text {graph }}$ follows from the the single-particle energy eigenstates $\eta_{\mathrm{nm}}^{\text {graph }}$, which are related to the single-particle eigenstates $\eta_{n m}$ of GaAs according to:

$$
\left|\eta_{n m}^{\text {graph }}\right\rangle=\left(\frac{1}{\sqrt{2}}\right)^{1-\delta_{|n|, 0}}\left(\begin{array}{c}
-\operatorname{sgn}(n) i\left|\eta_{|n|-1, m}\right\rangle \\
\left|\eta_{|n| m}\right\rangle
\end{array}\right) .
$$

Hence, if $n_{i}=0$ for $i=1,2,3,4$, then $V_{43,21}^{\text {graph }}=V_{43,21}$. Thus, the bare pseudopotentials for graphene in the $N=0$ lowest Landau level are identical to those in zero-thickness GaAs. In the $N^{\text {th }}$ Landau level, the pseudopotenials in graphene are a mixture of the $N^{\text {th }}$ and $(N-1)^{\text {th }}$ zero-thickness GaAs Landau levels.

Furthermore, the Landau level index $N$ in graphene is not bounded from below by zero but instead spans $-\infty$ to $+\infty$. Hence, the sum in Eq. (11) is modified in the case of graphene to

$$
\sum=\sum_{m_{x}, m_{x^{\prime}}=0}^{\infty} \sum_{n_{x}, n_{x^{\prime}}=-\infty}^{\infty} \sum_{\gamma, \gamma^{\prime}=\downarrow, \uparrow} .
$$

Again the prime on the sums over $n_{x}$ and $n_{x^{\prime}}$ indicates that we do not include $n_{x}=N$ or $n_{x^{\prime}}=N$ in the sums.

Another difference between graphene and GaAs is the Landau level mixing parameters. The Landau level mixing parameter for graphene is $\tilde{\kappa}=\left(e^{2} / \epsilon \ell_{0}\right) /\left(\hbar v_{F} / \ell_{0}\right)=$ $e^{2} /\left(\epsilon v_{F} \hbar\right) \equiv \alpha_{g}$ where $\alpha_{g}$ is the graphene fine-structure constant. Notably, $\tilde{\kappa}$ is independent of magnetic field strength $B$. This is in stark contrast to GaAs where $\kappa \propto 1 / \sqrt{B}$, so that Landau level mixing can be ignored for sufficiently large $B$. The Landau level mixing parameter in graphene $\tilde{\kappa}$ is a constant that can only be adjusted by manipulating the dielectric constant $\epsilon$, the Fermi velocity $v_{F}$, or $\hbar$ ! In freestanding (i.e., suspended) graphene $\tilde{\kappa} \approx 2.2^{49} \mid 51$, while for graphene on substrates it is $\tilde{\kappa} \approx 0.9$ for a $\mathrm{SiO}_{2}$ substrate ${ }^{49}$ and $\tilde{\kappa} \approx 0.5-0.8$ for a boron nitride substrate ${ }^{\sqrt{52182}}$ Thus, by adjusting the dielectric constant of the substrate from high dielectric to vacuum, one can only tune the Landau level mixing parameter between $0<\tilde{\kappa} \leq 2.2$ but it cannot be contin- uously tuned within a given device by tuning an experimental "knob" such as the magnetic field strength.

We also emphasize that since the spacing between Landau levels varies, unlike in GaAs where it is constant, and we expect the effect of Landau level mixing will depend on the Landau level in a manner that varies approximately as $\propto \tilde{\kappa} \sqrt{N}$.

\section{THE EFFECTIVE TWO- AND THREE-BODY PSEUDOPOTENTIALS FOR GRAPHENE}

We first investigate the three-body pseudopotentials in graphene since the discussion will help facilitate the later discussion of the two-body terms.

The most striking feature is that the three-body pseudopotentials vanish for $N=0$. Recall the expression for the three-body term generated in the perturbative expansion of the action for GaAs (Eq. (20)). For clarity, we write only the sum over Landau level index $n_{x}$ (and suppress the sums over $m_{x}$, spin, and cyclic permutations 
since they are not relevant for this argument)

$$
\begin{aligned}
& \sum_{n_{x}=-\infty, n_{x} \neq 0}^{\infty} \frac{\tilde{V}_{6 x, 21}^{\alpha^{\prime} \lambda, \beta \alpha} \tilde{V}_{54, x 3}^{\beta^{\prime} \gamma^{\prime}, \lambda \gamma}}{\tilde{E}_{n_{x}}^{\text {graph }}} \\
= & \frac{\ell_{0}}{\hbar v_{F}} \sum_{n_{x}=-\infty, n_{x} \neq 0}^{\infty} \frac{\tilde{V}_{6 x, 21}^{\alpha^{\prime} \lambda, \beta \alpha} \tilde{V}_{54, x 3}^{\beta^{\prime} \gamma^{\prime}, \lambda \gamma}}{\operatorname{sgn}\left(n_{x}\right) \sqrt{2\left|n_{x}\right|}} \\
= & \frac{\ell_{0}}{\hbar v_{F}} \sum_{n_{x}=-\infty}^{1} \frac{\tilde{V}_{6 x, 21}^{\alpha^{\prime} \lambda, \beta \alpha} \tilde{V}_{54, x 3}^{\beta^{\prime} \gamma^{\prime}, \lambda \gamma}}{-\sqrt{2\left|n_{x}\right|}}+\sum_{n_{x}=1}^{\infty} \frac{\tilde{V}_{6 x, 21}^{\alpha^{\prime} \lambda, \beta \alpha} \tilde{V}_{54, x 3}^{\beta^{\prime} \gamma^{\prime}, \lambda \gamma}}{\sqrt{2\left|n_{x}\right|}} \\
= & \frac{\ell_{0}}{\hbar v_{F}} \sum_{n_{x}=1}^{\infty}\left(\frac{\tilde{V}_{6 x, 21}^{\alpha^{\prime} \lambda, \beta \alpha} \tilde{V}_{54, x 3}^{\beta^{\prime} \gamma^{\prime}, \lambda \gamma}}{\sqrt{2\left|n_{x}\right|}}-\frac{\tilde{V}_{6 x, 21}^{\alpha^{\prime} \lambda, \beta \alpha} \tilde{V}_{54, x 3}^{\beta^{\prime} \gamma^{\prime}, \lambda \gamma}}{\sqrt{2\left|n_{x}\right|}}\right) \\
= & 0
\end{aligned}
$$

where $\tilde{V}_{43,21}^{\beta^{\prime} \alpha^{\prime}, \beta \alpha}=V_{43,21}^{\text {graph }} \delta^{\alpha \alpha^{\prime}} \delta^{\beta \beta^{\prime}}-V_{34,21}^{\text {graph }} \delta^{\alpha \beta^{\prime}} \delta^{\beta \alpha^{\prime}}$ and $\tilde{E}_{n_{x}}^{\text {graph }}=E_{n_{x}}^{\text {graph }}-\mu=\left(\hbar v_{F} / \ell_{0}\right)\left(\operatorname{sgn}\left(n_{x}\right) \sqrt{2\left|n_{x}\right|}-\right.$ $\operatorname{sgn}(N) \sqrt{2|N|})$. The canceling of the two terms in the second to last line above can be seen by going back to the definition of $V_{43,21}^{\text {graph }}$. Thus, the three-body terms exactly vanish in the lowest Landau level $(N=0)$. In the $N=0$ Landau level in graphene, particle-hole symmetry is an exact symmetry (in the absence of disorder) because there are as many Landau levels below $N=0$ as above. Consequently, Landau-level mixing cannot generate a three-body term because it would violate particlehole symmetry.

Outside of the lowest Landau level $(N \neq 0)$, the threebody pseudopotentials are non-zero. They are equal in magnitude and opposite in sign in the $N^{\text {th }}$ Landau level compared to the $-N^{\text {th }}$ Landau level (see Fig. 9). This, again, follows from particle-hole symmetry. Furthermore, they are, in general, negative for $N>0$ and, therefore, positive for $N<0$.

In fact, the Hamiltonian for graphene is invariant under the combined action of sublattice symmetry, timereversal, and charge conjugation. The sublattice symmetry takes $c \rightarrow-c$ on one sublattice, thereby inverting the kinetic energy in the absence of a magnetic field. Chargeconjugation transforms particles into holes, thereby inverting the kinetic energy in the absence of a magnetic field but leaving the Coulomb interaction energy unchanged (up to a shift of the chemical potential). Timereversal inverts the direction of the magnetic field. Therefore, the combination of all three transformations leaves both the kinetic and Coulomb energies unchanged. The combination transforms electrons in the $N^{\text {th }}$ Landau level into holes in the $-N^{\text {th }}$ Landau level. Therefore, we only give $N \geq 0$ results. The $N<0$ values may be obtained by symmetry, as described above.

Quantitatively, the three-body terms in graphene are similar in magnitude to those in GaAs, small compared to the bare two-body pseudopotentials, and decreasing in magnitude with increasing $M$. For $|N|=1$ there is nontrivial, non-monotonic $M$-dependence, where the pseudopotentials for $M=5,6$, and 7 are opposite in sign to those for $M=1,2,3$, and 8 . Table III lists the numerical
TABLE III: We list the numerical values of the three-body pseudopotentials $\tilde{V}_{M}^{(3)}(N)$ as a function of $M$ for graphene. All energies are given in units of $e^{2} / \epsilon \ell_{0}$.

\begin{tabular}{lrrrrr}
\hline \hline$N=$ & 0 & 1 & 2 & 3 & 4 \\
\hline$\tilde{V}_{1}^{(3)}(N)$ & 0 & -0.1237 & -0.0600 & -0.0377 & -0.0272 \\
$\tilde{V}_{2}^{(3)}(N)$ & 0 & -0.0856 & -0.0556 & -0.0363 & -0.0271 \\
$\tilde{V}_{3}^{(3)}(N)$ & 0 & -0.0537 & -0.0413 & -0.0266 & -0.0194 \\
$\tilde{V}_{5}^{(3)}(N)$ & 0 & 0.0135 & -0.0225 & -0.0221 & -0.0165 \\
$\tilde{V}_{6}^{(3)}(N)$ & 0 & 0.0313 & -0.0262 & -0.0225 & -0.0164 \\
$\tilde{V}_{7}^{(3)}(N)$ & 0 & 0.0205 & -0.0105 & -0.0133 & -0.0141 \\
$\tilde{V}_{8}^{(3)}(N)$ & 0 & -0.0123 & 0.0027 & -0.0148 & -0.0147 \\
\hline \hline
\end{tabular}

TABLE IV: We list the numerical values of $\delta \tilde{V}_{M}^{(2)}(N)$ for $N=0,1$, and 2 . The two-body correction consists of two contributions: one is a direction calculation of the ZS, ZS', and BCS diagrams and the other arises from the normal ordering of the three-body term [Eq. 27], please see the discussion in the text above. In the $N=0$ Landau level, these corrections are similar in magnitude to the results for GaAs. However, for $N=1$ and 2, the corrections are much larger and, in fact, it is expected that effective Landau level mixing parameter $\tilde{\kappa}$ is really $\propto \tilde{\kappa} \sqrt{N}$, see discussion in text. We use the $N=0$ values as the first term contribution for all $N$ since this term is so much smaller in magnitude than the second term and expected to make little difference to the final values or the physics. All energies are given in units of $e^{2} / \epsilon \ell_{0}$.

\begin{tabular}{lcrr}
\hline \hline & $N=0$ & 1 & 2 \\
\hline$\delta \tilde{V}_{0}^{(2)}(N)$ & -0.2638 & -0.4519 & -0.8529 \\
$\delta \tilde{V}_{1}^{(2)}(N)$ & -0.0633 & -0.1762 & -0.5240 \\
$\delta \tilde{V}_{2}^{(2)}(N)$ & -0.0407 & -0.0690 & -0.3943 \\
$\delta \tilde{V}_{3}^{(2)}(N)$ & -0.0143 & -0.0290 & -0.3010 \\
$\delta \tilde{V}_{4}^{(2)}(N)$ & -0.0090 & -0.0123 & -0.2179 \\
$\delta \tilde{V}_{5}^{(2)}(N)$ & -0.0052 & -0.0066 & -0.1510 \\
$\delta \tilde{V}_{6}^{(2)}(N)$ & -0.0034 & -0.0032 & -0.0996 \\
$\delta \tilde{V}_{7}^{(2)}(N)$ & -0.0030 & -0.0022 & -0.0643 \\
$\delta \tilde{V}_{8}^{(2)}(N)$ & -0.0022 & -0.0012 & -0.0400 \\
$\delta \tilde{V}_{9}^{(2)}(N)$ & -0.0016 & -0.0005 & -0.0242 \\
\hline \hline
\end{tabular}

values of $\tilde{V}_{M}^{(3)}(N)$ for $|N|=0-4$ and $M=1-3$, and $5-8$.

Turning now to the two-body pseudopotentials, we note first that because the energy increases with Landau level index $n$ as $\sqrt{n}$, the contribution from higher levels will be less strongly suppressed. In fact, unlike in the case of GaAs, it is extremely helpful to use the constraint following from angular momentum conservation to eliminate one of the Landau level index summations in Eqs. 14)-(16), thereby making the convergence of the sum more apparent.

The contribution to the two-body term coming from the normal ordering is usually an order of magnitude larger than the term coming from the $\mathrm{ZS}, \mathrm{ZS}$ ', and BCS 


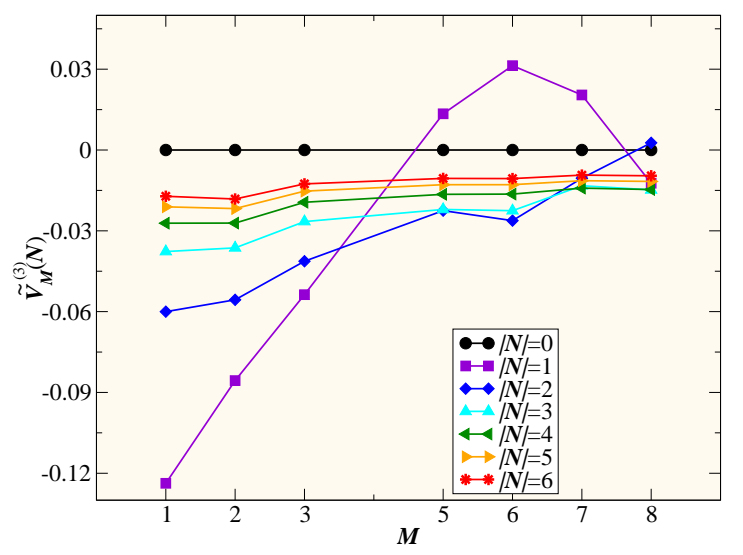

FIG. 9: (Color online) $\tilde{V}_{M}^{(3)}(N)$ as a function of $M$ for $N=0-6$. Note that for $N=0$ all three-body pseudopotentials exactly vanish. All energies are given in units of $e^{2} / \epsilon \ell_{0}$.

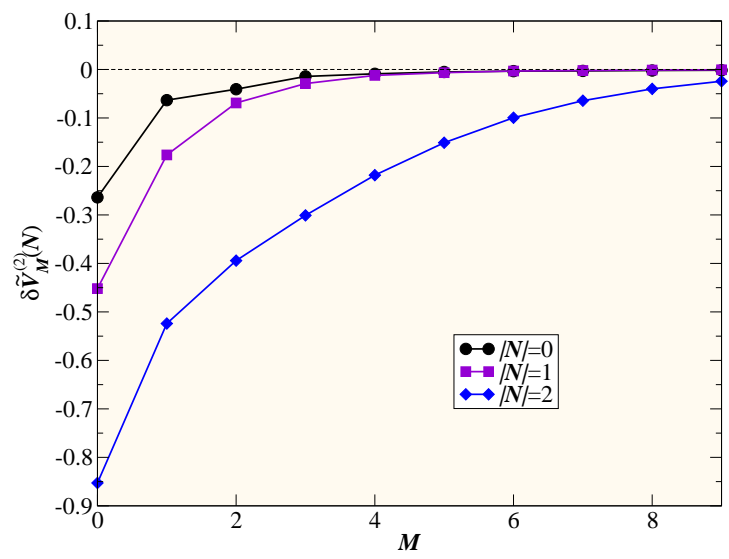

FIG. 10: (Color online) The two-body pseudopotential corrections, $\delta \tilde{V}_{M}^{(2)}(N)$ for $N=0,1$, and 2 for graphene as a function of $M$. It is clear from this figure that the pseudopotential corrections increase for increasing Landau level $N$ reflecting the conjecture that the effective Landau level mixing parameter is actually $\propto \tilde{\kappa} \sqrt{N}$ and not simply $\tilde{\kappa}$. All energies are given in units of $e^{2} / \epsilon \ell_{0}$.

diagrams alone. Because of this fact, and the fact that finding the convergence of the smaller two-body terms is laborious, we use the $N=0$ values of the two-body terms for all Landau levels as an upper limit and good approximation. Table IV lists the numerical values of $V_{M}^{(2)}(N)$.

In addition, in Fig. 10 we plot the Landau level mixing induced corrections to the two-body pseudopotentials as a function of relative angular momentum $M$ for $N=0,1$, and 2 . The two-body corrections are universally negative and for small $M$ can be approximately half the value of the bare pseudopotential. Interestingly, the corrections become larger in higher Landau levels owing to the fact that the effective Landau level mixing parameter is $\propto$ $\tilde{\kappa} \sqrt{N}$.

In Fig. 11 we show the two- and three-body pseudopotentials as a function of both $\tilde{\kappa}$ and $M$. For $N=0$, Landau level mixing has little effect on the pseudopotentials, i.e., the corrections to the two-body term are relatively small and there is no three-body term-this might help explain why the FQHE is observed in the $N=0$ Landau level in graphene, when the system is spin and valley polarized, even though Landau level mixing cannot naively be ignored due to a large value of $\tilde{\kappa}$. This further underscores the fact that the FQHE in graphene in the $N=0$ is observed to be, and is expected to be, nearly identical to that of GaAs (other than interesting complications that arise when the spin and valley degeneracies remain). However, for $N \neq 0$, there are strong corrections in the form of negative two-body corrections and emergent three-body terms. For moderate values of $\tilde{\kappa}$ it is not at all clear that graphene would even exhibit the FQHE according to our calculations-the two-body terms can be of the same order of magnitude as the three-body terms. We also emphasize that with such strong Landau level mixing corrections to first order in $\tilde{\kappa}$ it is possible that higher order corrections will be significant, however, it is likely that these corrections (which we are ignoring) will make the situation worse for the FQHE, not better.

Of course, it remains to be seen theoretically what effect the three-body terms have on the FQHE. Exact diagonalization studies using the above effective Hamiltonian are likely to shed light on this problem $\frac{81}{\text {. }}$

\section{CONCLUSION}

Quantum electrodynamics is a paradigm of a perturbative theory: the dimensionless expansion parameter $\alpha \approx 1 / 137$ is small, so effects that occur at each order in perturbation theory are successively less important than those that occurred at the previous order. However, in condensed matter physics, we have grown accustomed to perturbation theory in dimensionless parameters that are not particularly small. Landau parameters are typically $\mathcal{O}(1)$, yet Fermi liquid theory remains valid for ${ }^{3} \mathrm{He}$ and, often, for electrons in metals. The ratio of the Coulomb energy to the kinetic energy in a metal, $r_{s}$, can be moderately large, without the metallic state being destabilized (in favor of, say, a Wigner crystal). But we should guard against the possibility that we have been lulled into a false sense of security. There is no quantum Hall experiment in which Landau-level mixing is obviously negligible a priori. In the original observation of the fractional quantum Hall effect at $\nu=1 / 3$, the Landau level mixing parameter is $\kappa=0.65$. This might be small enough that one can reach this value via an expansion in powers of $\kappa$ about $\kappa=0$ or it might not, but it is certainly not a very small number such as $1 / 137$ which one a priori expects to be negligible. In the $N=1$ Landau level, $1<\kappa<2.5$, so it is even less clear that Landau level mixing is small. 


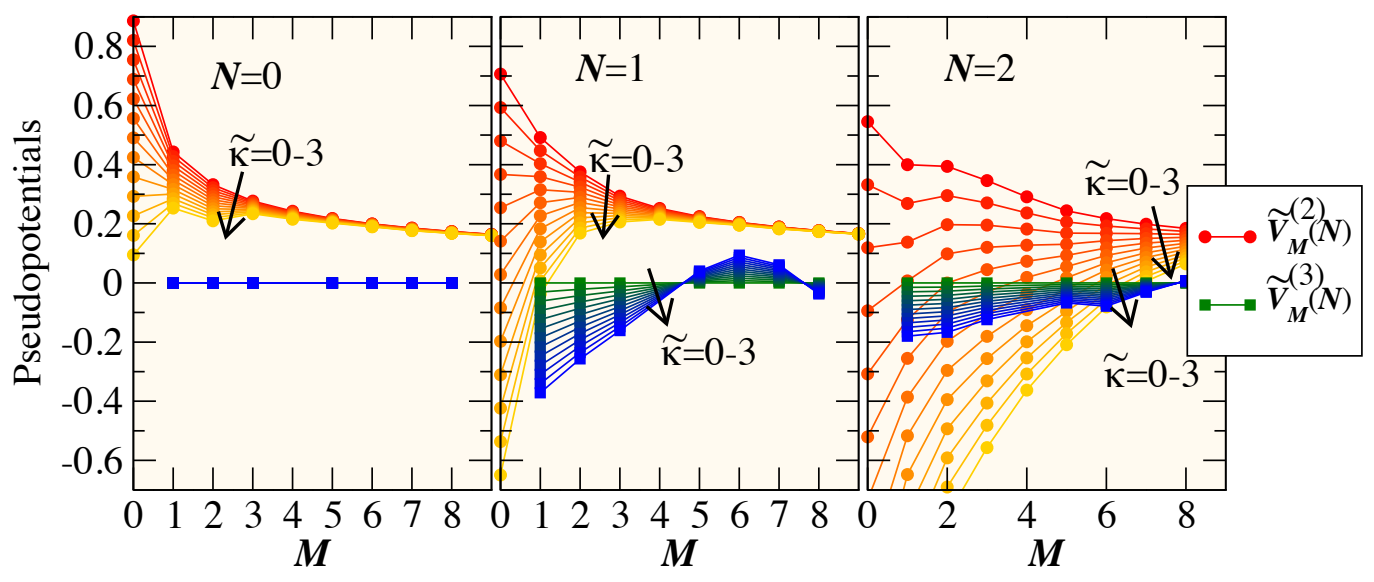

FIG. 11: (Color online) Both the two- and three-body pseudopotentials as functions of $M$ for $\tilde{\kappa}=0-3$ for graphene, in the $N=0,1$, and 2 Landau levels. For the lowest Landau level $(N=0)$ there is no three-body term (it exactly vanishes) and the two-body corrections are modest. Hence, one would expect, from merely studying the pseudopotentials, that the FQHE in $N=0$ Landau level for graphene would be nearly identical to that of GaAs. However, for $N \neq 0$, we observe significant corrections to the two-body pseudopotentials and significant three-body terms. It is not at all clear what these effects will have on the FQHE. Naively, one is tempted to suggest that the FQHE will not be observed in $N \neq 0$ Landau levels in graphene, or if it is observed, it will be of an exotic variety. All energies are given in units of $e^{2} / \epsilon \ell_{0}$.

In the case of graphene, $0.5<\tilde{\kappa}<2.2$, so the situation is no better.

The coefficient of $O(\kappa)$ corrections to two- and threebody pseudopotentials due to Landau level mixing are almost always numerically small. Consequently, these corrections are small even at $\kappa=1$. (Most of the corrections are small even at $\kappa=10$.) Since we have not computed the order $\kappa^{2}$ corrections, this does not prove that experiments are effectively in a small $\kappa$ regime. However, it does, at least, raise this possibility - or, in other words, the possibility that the true expansion parameter is $\sim \kappa / 10$.

Of course, for the FQHE in the $N \neq 0$ Landau level of graphene, the corrections are not necessarily small and it is very possible that a perturbative treatment of Landau level mixing is inappropriate.

Strictly speaking, our computation yields an effective action, rather than an effective Hamiltonian - as is always the case when integrating out high-energy states to produce a low-energy effective theory. This effective action has retarded interactions but, at lowest-order in $\kappa$, these retarded interactions can be neglected. Moreover, our calculation assumed fixed chemical potential. At lowest-order in $\kappa$, we can simply take this to be the Landau level energy $\hbar \omega_{c}\left(N+\frac{1}{2}\right)$ or $\operatorname{sgn}(N) \sqrt{2|N|} \hbar v_{F} / \ell_{0}$, regardless of filling fraction within the $N^{\text {th }}$ Landau level. However, in going to higher-order in $\kappa$, we would have to tune the chemical potential to determine the filling fraction. For these two reasons, it will be difficult to determine the ground state of the system to order $\kappa^{2}$. But it should still be possible to derive an effective action (albeit with retarded interactions) from which it would be possible to determine if order $\kappa^{2}$ effects are also small even at $\kappa=1$.

We note that, for large numbers of electrons, fixing the chemical potential will be the same as fixing the electron number (or, equivalently, the filling fraction). However, for the small numbers of electrons considered in numerical exact diagonalization studies, fixed chemical potential and fixed electron number are not the same. For fixed chemical potential, $\Delta n \propto \sqrt{n}$ (where $n$ is the electron number) which can be large. Thus, our effective Hamiltonian is only, strictly speaking, correct for systems with a large number of electrons, not a small number. However, if the goal of studying small systems is to extrapolate their properties to larger ones to determine the solution in the thermodynamic limit with fixed filling fraction, then our effective Hamiltonian can be used.

Even if it is quantitatively small, Landau-level mixing could, nevertheless, have significant qualitative effects. It is the leading effect that breaks particle-hole symmetry within a Landau level (except for the $N=0$ Landau level in graphene, where this is an exact symmetry). This has important effects at $\nu=5 / 2$ in GaAs, where it could break the degeneracy between the MooreRead Pfaffian state and the anti-Pfaffian state, as we discuss elsewhere ${ }^{81}$. It can also have important effects at $\nu=7 / 3,8 / 3$ and $12 / 5,13 / 5$-with particle symmetry broken, these states may not be particle-hole conjugates of each other after all but, instead, have very different characters. Indeed, even within the lowest Landau level, there are obvious differences between particle-hole conjugate plateaus, such as $\nu=1 / 5$ and $\nu=4 / 5$. Presumably the underlying cause is Landau level mixing.

Finally, we note that, although the corrections to the effective Hamiltonian due to Landau level mixing are 
small compared to the bare terms, they are not small compared to the energy gaps of quantum Hall states. So it is possible, in principle, for three-body terms to have a non-negligible effect on the energy gaps of some fractional quantum Hall states while, at the same time, order $\kappa^{2}$ terms can be neglected. In such a case, our order $\kappa$ effective Hamiltonian would be the most promising starting point for an attempt to make a direct quantitative comparison between experiments and numerical simulation of quantum Hall systems.

Note: Recently, we received a first draft of a paper by Sodemann and MacDonald ${ }^{84}$ containing some results on the effect of Landau level mixing on the FQHE in GaAs in the zero-thickness limit. They reproduced the $d / \ell_{0}=0$ limit of our three-body interaction (which, in turn, is equal to the three-body interaction of Bishara and Nayak ${ }^{59}$ ) and reproduced the $d / \ell_{0}=0$ limit of our two-body interaction in the $N=0$ Landau level. However our two-body interaction in the $N=1$ Landau level diverges from theirs. In contrast, the $d / \ell_{0}=0$ limit of our two- and three-body interactions in both the $N=0$ and $N=1$ Landau levels are within a few percent of the results of Rezayi and Simori 83 .

\section{Acknowledgments}

We acknowledge DARPA QuEST and Microsoft for funding and M. R. P. acknowledges California State University Long Beach Start-up Funds. We acknowledge helpful discussions with Matthias Troyer and Matt Hastings and, additionally, Euyheon Hwang, Shaffique Adam, and Sankar Das Sarma regarding the effective dielectric constant for graphene on various substrates. We benefited from stimulating and helpful discussions with Steve Simon, Ed Rezayi, and Inti Sodemann. We are especially grateful to Simon and Rezayi for sharing their, at the time, unpublished results 83 . We thank the Aspen Center for Physics, where part of this work was completed, for its hospitality. Last, we thank one of the referees for helpful comments that improved the manuscript.
1 A. Y. Kitaev, Ann. Phys. (N.Y.) 303, 2 (2003).

2 S. Das Sarma, M. Freedman, and C. Nayak, Phys. Rev. Lett. 94, 166802 (2005).

3 C. Nayak, S. H. Simon, A. Stern, M. Freedman, and S. Das Sarma, Rev. Mod. Phys. 80, 1083 (2008).

${ }^{4}$ R. Willett, J. P. Eisenstein, H. L. Stormer, D. C. Tsui, A. C. Gossard, and J. H. English, Phys. Rev. Lett. 59, 1776 (1987).

${ }^{5}$ W. Pan, J.-S. Xia, V. Shvarts, D. E. Adams, H. L. Stormer, D. C. Tsui, L. N. Pfieffer, K. W. Baldwin, and K. W. West, Phys. Rev. Lett. 83, 3530 (1999).

${ }^{6}$ J. P. Eisenstein, K. B. Cooper, L. N. Pfeiffer, and K. W. West, Phys. Rev. Lett. 88, 076801 (2002).

7 I. Radu, J. Miller, C. Marcus, M. Kastner, L. N. Pfeiffer, and K. West, Science 320, 899 (2008).

8 M. Dolev, M. Heiblum, V. Umansky, A. Stern, and D. Mahalu, Nature 452, 829 (2008).

9 R. L. Willett, L. N. Pfeiffer, and K. W. West, Proceedings of the National Academy of Sciences (USA) 106, 8853 (2009).

10 M. Stern, P. Plochocka, V. Umansky, D. K. Maude, M. Potemski, and I. Bar-Joseph, Phys. Rev. Lett. 105, 096801 (2010).

11 A. Bid, N. Ofek, H. Inoue, M. Heiblum, C. L. Kane, V. Umansky, and D. Mahalu, Nature (London) 466, 585 (2010).

12 T. D. Rhone, J. Yan, Y. Gallais, A. Pinczuk, L. Pfeiffer, and K. West Phys. Rev. Lett. 106, 196805 (2011).

13 V. Venkatachalam, A. Yacoby, L. Pfeiffer, and K. West, Nature (London) 469, 185 (2011).

14 L. Tiemann, G. Gamez, N. Kumada, and K. Muraki, Science 335, 828 (2011).

15 G. Moore and N. Read, Nucl. Phys. B 360, 362 (1991).

16 S.-S. Lee, S. Ryu, C. Nayak, and M. P. A. Fisher, Phys. Rev. Lett. 99, 236807 (2007).

17 M. Levin, B. I. Halperin, and B. Rosenow, Phys. Rev. Lett. 99, 236806 (2007).
18 P. Bonderson, V. Gurarie, and C. Nayak, Phys. Rev. B 83, 075303 (2011).

19 C. Nayak and F. Wilczek, Nucl. Phys. B 479, 529 (1996).

20 N. Read and E. Rezayi, Phys. Rev. B 54, 16864 (1996).

21 V. Gurarie and C. Nayak, Nucl. Phys. B 506, 685 (1997).

${ }^{22}$ N. Read and D. Green, Phys. Rev. B 61, 10267 (2000).

23 D. A. Ivanov, Phys. Rev. Lett. 86, 268 (2001).

24 Y. Tserkovnyak and S. H. Simon, Phys. Rev. Lett. 90, 016802 (2003).

25 A. Stern, F. von Oppen, and E. Mariani, Phys. Rev. B 70, 205338 (2004).

${ }^{26}$ M. Stone and S.-B. Chung, Phys. Rev. B 73, 014505 (2006).

27 A. Seidel, Phys. Rev. Lett. 101, 196802 (2008).

28 N. Read, Phys. Rev. B 79, 045308 (2009), arXiv:0805.2507.

29 M. Baraban, G. Zikos, N. Bonesteel, and S. H. Simon, Phys. Rev. Lett. 103, 076801 (2009).

30 E. Prodan and F. D. M. Haldane, Phys. Rev. B 80, 115121 (2009).

31 R. H. Morf, Phys. Rev. Lett. 80, 1505 (1998).

32 E. H. Rezayi and F. D. M. Haldane, Phys. Rev. Lett. 84, 4685 (2000).

33 A. E. Feiguin, E. Rezayi, C. Nayak, and S. Das Sarma, Phys. Rev. Lett. 100, 166803 (2008).

${ }^{34}$ M. R. Peterson, T. Jolicoeur, and S. Das Sarma, Phys. Rev. Lett. 101, 016807 (2008).

35 M. R. Peterson, T. Jolicoeur, and S. Das Sarma, Phys. Rev. B 78, 155308 (2008).

36 M. R. Peterson, Journal of Physics: Conference Series 402, 012021 (2012).

37 E. H. Rezayi and S. H. Simon (2009), arXiv.org:0912.0109.

38 A. Wójs, C. Tőke, and J. K. Jain, Phys. Rev. Lett. 105, $096802(2010)$.

39 M. Storni, R. H. Morf, and S. Das Sarma, Phys. Rev. Lett. 104, 076803 (2010).

40 M. P. Lilly, K. B. Cooper, J. P. Eisenstein, L. N. Pfeiffer, and K. W. West, Phys. Rev. Lett. 82, 394 (1999). 
41 R. Du, D. Tsui, H. Stormer, L. Pfeiffer, K. Baldwin, and K. West, Solid State Communications 109, 389 (1999), arXiv:cond-mat/9812025.

42 R. L. Willett, R. R. Ruel, K. W. West, and L. N. Pfeiffer, Phys. Rev. Lett. 71, 3846 (1993).

${ }^{43}$ W. Pan, R. R. Du, H. L. Stormer, D. C. Tsui, L. N. Pfeiffer, K. W. Baldwin, and K. W. West, Phys. Rev. Lett. 83, 820 (1999).

44 M. P. Lilly, K. B. Cooper, J. P. Eisenstein, L. N. Pfeiffer, and K. W. West, Phys. Rev. Lett. 83, 824 (1999).

45 J. Xia, V. Cvicek, J. P. Eisenstein, L. N. Pfeiffer, and K. W. West, Phys. Rev. Lett. 105, 176807 (2010), 1006.2199.

46 One may also question whether the same ground state is observed in all experiments, given that the $\nu=5 / 2$ state is observed from $B=2 \mathrm{~T}$ all the way up to $B=12 \mathrm{~T}$ (which corresponds to the range $0.7<\kappa<1.8$ ) and is observed in both quantum wells and heterostructures - implying that the Hamiltonian varies considerably among the various experiments which have observed a $\nu=5 / 2$ plateau.

47 Most of these numerical studies also assume that the ground state is fully spin-polarized. The few which do not make such an assumption, in fact, make a strong case for a spin-polarized ground state $31|85| 86$. However, experiments indicate that the ground state may not be polarized

48 N. Samkharadze, L. N. Pfeiffer, K. W. West, and G. A. Csathy (2013), arXiv:1302.1444.

${ }^{49}$ K. I. Bolotin, F. Ghahari, M. D. Shulman, H. L. Stormer, and P. Kim, Nature 462, 196 (2009).

${ }^{50}$ F. Ghahari, Y. Zhao, P. Cadden-Zimansky, K. Bolotin, and P. Kim, Phys. Rev. Lett. 106, 046801 (2011).

51 X. Du, I. Skachko, F. Duerr, A. Luican, and E. Y. Andrei, Nature 462, 192 (2009).

52 C. R. Dean, A. F. Young, P. Cadden-Zimansky, L. Wang, H. Ren, K. Watanabe, T. Taniguchi, P. Kim, J. Hone, and K. L. Shepard, Nature Physics 7, 693 (2011).

${ }^{53}$ V. M. Apalkov and T. Chakraborty, Phys. Rev. Lett. 97, 126801 (2006)

${ }^{54}$ C. Tőke, P. E. Lammert, V. H. Crespi, and J. K. Jain, Phys. Rev. B 74, 235417 (2006).

55 C. Tőke and J. K. Jain, Phys. Rev. B 75, 245440 (2007).

56 M. O. Goerbig and N. Regnault, Phys. Rev. B 75, 241405 (2007).

57 Z. Papic, M. Goerbig, and N. Regnault, Solid State Communications 149, 1056 (2009), ISSN 0038-1098.

58 C. Tőke and J. K. Jain, Phys. Rev. B 76, 081403 (2007).

59 W. Bishara and C. Nayak, Phys. Rev. B 80, 121302 (2009).

60 S. Das Sarma, S. Adam, E. H. Hwang, and E. Rossi, Rev. Mod. Phys. 83, 407 (2011).

${ }^{61}$ Interferometry experiments ${ }^{7 / 9}$ can distinquish between a $\nu=5 / 2$ state described by the MR Pfaffian or antiPfaffian.

62 J. Nuebler, V. Umansky, R. Morf, M. Heiblum, K. von
Klitzing, and J. Smet, Phys. Rev. B 81, 035316 (2010).

63 E. H. Rezayi and F. D. M. Haldane, Phys. Rev. B 42, 4532 (1990).

64 R. Morf and N. d'Ambrumenil, Phys. Rev. B 68, 113309 (2003).

65 A. H. MacDonald and G. C. Aers, Phys. Rev. B 29, 5976 (1984).

66 S. He, F. C. Zhang, X. C. Xie, and S. Das Sarma, Phys. Rev. B 42, 11376 (1990).

67 F. Zhang and S. Das Sarma, Phys.Rev. B 33, 2903 (1986).

68 M. W. Ortalano, S. He, and S. Das Sarma, Phys. Rev. B 55, 7702 (1997).

69 K. Park and J. K. Jain, Phys. Rev. Lett. 81, 4200 (1998).

70 K. Park, N. Meskini, and J. K. Jain, Journal of Physics:Condensed Matter 11, 7283 (1999).

71 A. H. MacDonald, in Proceedings of the Les Houches Summer School on Mesocsopic Quantum Physics, edited by E. Akkermans, G. Montambaux, J. L. Pichard, and J. Zinn-Justin (Elsevier, Amsterdam, 1995).

72 J. Jain, Composite fermions (Cambridge University Press, 2007).

${ }^{73}$ Y. Liu, J. Shabani, D. Kamburov, M. Shayegan, L. N. Pfeiffer, K. W. West, and K. W. Baldwin, Phys. Rev. Lett. 107, 266802 (2011).

74 J. Nuebler, B. Friess, V. Umansky, B. Rosenow, M. Heiblum, K. von Klitzing, and J. Smet, Phys. Rev. Lett. 108, 046804 (2012).

75 R. Shankar, Rev. Mod. Phys. 66, 129 (1994).

76 W. Bishara (2009), CalTech Ph.D. Thesis.

77 S. H. Simon, E. H. Rezayi, and N. R. Cooper, Phys. Rev. B 75, 195306 (2007).

78 The corrections for $d / \ell_{0}=0$ were recently also reported in Ref. 84 for the $N=0$ and 1 Landau levels. Their numbers agree with ours in the $N=0$ Landau level but not the $N=1$.

79 M. Greiter, X.-G. Wen, and F. Wilczek, Phys. Rev. Lett. 66, 3205 (1991).

80 M. R. Peterson, K. Park, and S. Das Sarma, Phys. Rev. Lett. 101, 156803 (2008).

81 M. R. Peterson and C. Nayak, unpublished.

82 S. Das Sarma and E. H. Hwang, Phys. Rev. B 83, 121405 (2011).

83 S. H. Simon and E. H. Rezayi, Phys. Rev. B 87, 155426 (2013)

84 I. Sodemann and A. H. MacDonald (2013), arXiv:1302.3986.

85 I. Dimov, B. I. Halperin, and C. Nayak, Phys. Rev. Lett. 100, 126804 (2008).

${ }^{86}$ A. E. Feiguin, E. Rezayi, K. Yang, C. Nayak, and S. Das Sarma, Phys. Rev. B 79, 115322 (2009). 